\title{
1 Antiviral and Neuroprotective Abilities of Influenza Virus Infection in Tractable Brain
}

\section{Organoids}

5 Pharmaceutical Sciences, Peking University, Beijing, China.

6 These authors contributed equally to this work.

7 "Corresponding: xqing@hsc.pku.edu.cn 


\section{Abstract}

10 Human pluripotent stem cell (hPSC)-derived brain organoids offer an unprecedented opportunity for various

11 applications as an in vitro model, such as modeling virus infection and drug screening. In this study, we

12 present an experimental brain organoid platform for modeling infection with multiple viruses (e.g., influenza

13 virus or enterovirus). Brain organoids challenged by influenza viruses (H1N1-WSN and H3N2-HKT68) had

14 decreased overall organoid size, similar to ZIKA virus infection, while enteroviruses (EV68 and EV71)

15 infected brain organoids displayed the opposite result. Then, we studied the molecular events in WSN-infected

16 organoids, and we found that WSN could widely infect multiple cell types, and preferentially infected MAP2+

17 neurons compared to SOX2+ neural stem cells (NSCs) and GFAP+ astrocytes in brain organoids, and induced

18 apoptosis of NSCs and neurons, but not astrocytes. The inflammatory responses in organoids observed to

19 occur (Tumor necrosis factor alpha, interferon gamma, and interleukin 6) after WSN infection may further

20 facilitate brain damage. Furthermore, transcriptional profiling revealed several upregulated genes (CSAG3 and

$21 O A S 2)$ and downregulated genes (CDC2OB, KCNJ13, OTX2-AS1, CROCC2, and F5) after WSN infection for

2224 hpi and 96 hpi, implicating antiviral drugs development responses to WSN. Finally, we explored

23 neurotrophic factors (e.g., BDNF, GDNF, and NT3) and PYC-12 as antiviral and neuroprotective reagents,

24 which could significantly suppress virus infection, apoptosis, and inflammatory responses. Collectively, we

25 established a tractable experimental model system to investigate the impact and mechanism of virus infection

26 on human brain development, and provide a platform for rapidly screening therapeutic compounds, advancing

27 the development of antiviral strategies.

28 Keywords: Influenza virus; Brain organoids; Human pluripotent stem cells; RNA transcriptomic profiling; 
bioRxiv preprint doi: https://doi.org/10.1101/2022.03.02.482634; this version posted March 2, 2022. The copyright holder for this preprint (which was not certified by peer review) is the author/funder. All rights reserved. No reuse allowed without permission.

\section{Antiviral screening.}




\section{Introduction}

32 Central nervous system (CNS) infections are one of the most critical public health problems, and they lead to 33 high morbidity and mortality every year, predominately in young children, the elderly, and the

34 immunocompromised (Englund et al., 2011; Keipp Talbot and Falsey, 2010; Tregoning and Schwarze, 2010).

35 CNS infections often lead to neurological sequelae, including epilepsy (Vehapoglu et al., 2015), and 36 neurodevelopmental disorders, such as attention-deficit/hyperactivity disorder (ADHD) (Hoekstra, 2019) and 37 autism spectrum disorder (ASD) (Sauer et al., 2021). Some known neurotropic viruses, such as measles virus 38 (MV), herpes virus, and human immunodeficiency virus (HIV), can cause CNS infections (Koyuncu et al., 39 2013). Moreover, respiratory viruses including human respiratory syncytial virus (HRSV), influenza virus 40 (IV), and coronavirus (Cov) have also become key factors responsible for CNS pathologies. In particular, 41 COVID-19, which is caused by severe acute respiratory syndrome coronavirus 2 (SARS-CoV-2), has been 42 characterized by respiratory failure in critically ill patients. The outbreak of COVID-19 has led to a pandemic, 43 and serious and even fatal manifestations have been seen in the brain (Johansson et al., 2020). The annual 44 pandemic of influenza A virus (IAV) has been shown to cause neurodegenerative diseases in clinical 45 investigations (McGavern and Kang, 2011). IAV is often the causative agent of upper respiratory infection by 46 recognizing sialic acids (SA) receptor in humans affecting people of all ages (Han et al., 2018). Although the 47 influenza virus primarily infects the lungs, its neuropathological effects have also been shown in the clinic, 48 including febrile seizures, myelitis, focal encephalitis, and even meningitis (Liang et al., 2018). However, the 49 neuropathogenesis of these manifestations remains elusive, owing to the lack of physiological in vitro models 
50 instead of patient-derived brain tissues. Therefore, there is an urgent need of in vitro physiological models of

51 virus infection to identify the neuropathological mechanism in the brain.

52 Human pluripotent stem cell (hPSC)-derived organoids, which are an in vitro self-organizing and 53 self-renewing three-dimensional tissue recapitulating the cytoarchitecture and functional components of 54 human tissue, can be amendable to tissue development, disease modeling, drug screening, and therapeutic 55 discovery (De Crignis et al., 2021; Qian et al., 2019). For example, Chen et al. (2021) generated 56 serum-exposed brain organoids that were able to recapitulate Alzheimer's disease (AD)-like pathologies, 57 providing a powerful platform for both mechanistic study and therapeutic development of AD treatments. Dang et al. (2016) utilized human cerebral organoids to demonstrate that Zika virus (ZIKV) could decrease the 59 number of neural progenitors through activation of the innate immune receptor TLR3, resulting in dysregulation of a network of genes involved in axon guidance, neurogenesis, differentiation, and apoptosis.

61 In addition, Clevers' group leveraged colorectal cancer (CRC) organoid lines to screen target-known inhibitors 62 and chemotherapy drugs (Van De Wetering et al., 2015), and also used breast cancer organoid lines to screen 63 EGFR/AKT/mTORC pathway inhibitors (Sachs et al., 2018). Currently, several organoids (e.g., lung, liver, 64 gut, brain, and kidney) mimicking different tissues have been used to study the effects of SARS-CoV-2 on 65 tissues in vitro, and tissue histopathology ( $\mathrm{Yu}, 2021)$. The advantage of brain organoids over cell lines is that 66 organoids model the three-dimensional organ to achieve multiple cellular interactions, and rather than animal 67 models, the use of organoids enables studies of humans. Therefore, organoid technology offers a significant 68 advantage in terms of mimicking in vivo tissue structures for testing antiviral compounds. To date, influenza 69 virus-infected brain organoid use to identify neuropathogenesis or for drug screening has not been reported. 
70 In this study, we evaluated the potential of brain organoids as in vitro infection models, and studied the

71 neuropathogenesis of brain organoids challenged by WSN using hPSC-derived brain organoids. We found that

72 WSN preferentially infected MAP2+ neurons in brain organoids, and further damaged the brain by eliciting

73 inflammatory factor release (TNF- $\alpha, \mathrm{INF}-\gamma$, and IL-6), as well as inducing the apoptosis of NSCs and neurons,

74 but not astrocytes. Additionally, RNA transcriptomic profiles revealed new protein targets and noncoding

75 RNAs for the development of antiviral strategies. Finally, we conducted an antiviral study and found that

76 some specific neurotrophic factors (e.g., BDNF, GDNF, and NT3) and a potential antiviral drug candidate,

77 PYC-12, could both significantly decrease virus replication to achieve antiviral and neuroprotective effects.

78 Collectively, human organoids were shown to serve as an invaluable tool in the field of virus research to

79 investigate the molecular mechanisms underlying virus replication and antiviral drug screening. 


\section{Results}

82 Generation and characterization of cerebral organoids from hPSCs

83 To study the neuropathogenesis of virus-infected brain organoids instead of patient-derived brain tissue, we

84 first generated human pluripotent stem cell (hPSC)-derived brain organoids as previously described (Lancaster 85 and Knoblich, 2014), with slight modifications. Briefly, hPSCs were first dissociated into single cells, 86 followed by centrifugation at $100 \mathrm{~g}$ for 3 minutes. hPSCs were allowed to form embryoid bodies (EBs) in 87 Essential 8 medium containing $10 \mu \mathrm{M}$ ROCK inhibitor for 4 days, incubated in neural induction medium to 88 develop neuroectoderm, and then transferred to a Matrigel droplet. Finally, these Matrigel droplet 89 encapsulated tissues were transferred to a spinning bioreactor, which promoted nutrient and oxygen exchange 90 to further develop into brain organoids (Fig. 1A). The representative bright field images display the entire 91 development of brain organoids at indicated time points (Fig. 1B). In the process of organoid development, 92 the diameter $(\mu \mathrm{m})$ of organoids increased, while the area $\left(\mu \mathrm{m}^{2}\right)$ increased in early stages and decreased 93 obviously in later stages (Fig. 1C, D). Next, we performed immunostaining of brain organoids at days 15, 30, 94 60, and 120 with the neural stem cell marker-SOX2 and the neuron marker-MAP2 (Fig. 1E, F). Statistical 95 analysis showed that the majority of the cells on day 15 and day 30 organoids were neural stem cells (SOX2+), 96 while the majority of the cells on day 60 and day 120 organoids were neurons (MAP2+) (Fig. 1G). Finally, 97 quantitative real-time polymerase chain reaction (RT-PCR) confirmed the specific markers in the entire 98 process of brain organoid development, including the hPSC markers, OCT4 and NANOG; the NSC marker, 99 PAX6; the forebrain markers, FOXG1 and SIX3; the hindbrain markers, KROX20 and ISL1; the neuron marker, 00 TUJ1; and the deep-layer cortical neuron markers, CTIP2 and TBR1 (Fig. 1H). In summary, these results 
01 suggested that there was robust differentiation of brain organoids and following applications of virus infection

02 model.

03 Brain organoid as an in vitro model for distinct virus infection

04 To model the effects of virus infection on early human brain development, we used hPSC-derived brain 05 organoids at day 40 of differentiation. We challenged these day 40 brain organoids with a diverse panel of 06 viruses, including influenza viruses (H1N1-WSN and H3N2-HKT68), enteroviruses (EV68 and EV71) and 07 severe fever with thrombocytopenia syndrome virus (SFTSV) at indicated timepoints, and identified the 08 optimal doses for virus infection. These were $1 \times 10^{6} \mathrm{pfu}$ for WSN, $5 \times 10^{6} \mathrm{pfu}$ for H3N2, 8x10 $0^{4}$ fu for EV68, 09 and $4 \times 10^{6}$ pfu for EV71 (Fig. 2A). The representative bright field images show a decrease in overall organoid 10 size in the influenza virus infection group at indicated virus doses (Fig. 2B, D), which was similar to Zika 11 virus infection but not as severe (Tang et al., 2016). Statistical analysis of diameters $(\mu \mathrm{m})$ and $\operatorname{areas}\left(\mu \mathrm{m}^{2}\right)$ for 12 influenza virus-infected organoids showed a dose-dependent size decrease (Fig. 2C, E). Surprisingly, when 13 we challenged day 40 organoids with the other two H1N1 subtypes, PR8 and CA07, we observed a slight 14 increase of organoid size at indicated virus doses through representative bright field images and statistical 15 analysis of diameters $(\mu \mathrm{m})$ and areas $\left(\mu \mathrm{m}^{2}\right)$ (Fig. S1). Meanwhile, enteroviruses (EV68 and EV71)-infected 16 brain organoids resulted in a significant increase of overall organoid size at indicated virus doses through 17 bright field images and statistical analysis of their diameters and areas (Fig. 2F-I). However, SFTSV-infected 18 brain organoids showed no obvious changes in overall organoid size (Fig. 2J, K). Therefore, the 19 morphological changes of organoids challenged by multiple viruses may be different due to different 20 mechanisms. 
21 Next, to better understand the infection mechanism of brain organoids with virus, we primarily focused on the

22 study of WSN infected brain organoids. These brain organoids were generated as mentioned previously and

23 infected on days 30, 60, and 250 (Fig. 3A). Before WSN infection of organoids, we first confirmed that $\sim 20 \%$

24 NESTIN+ NSCs derived from hPSCs and $\sim 10 \%$ GFAP+ astrocyte could be infected by WSN at the cellular

25 level (Fig. 3B). The advantage of brain organoids over cell lines is that organoids model a three-dimensional

26 organ to achieve multiple cellular interactions, and the advantage of organoids over animal models is that

27 organoids enable studies of humans. Immunostaining of day 30 and day 60 brain organoids with SOX2+

28 NSCs and MAP2+ neurons showed a time-dependent increase of NP+ cells compared to mock infection,

29 especially for MAP2+ neurons (Fig. 3C, D). The statistical percentage of NP+ cells demonstrated that WSN

30 was more likely to infect MAP2+ neurons $(\sim 60 \%)$ at 4 dpi, while only $\sim 10 \%-20 \%$ of NSCs were infected at

311 dpi and 4 dpi, respectively (Fig. 3E, H). The intracellular and extracellular virus titers showed a

32 time-dependent increase after WNS infection in day 30 or day 60 organoids (Fig. 3F, G, I, J). To further

33 investigate long-term infection, we cultured brain organoids for 250 days. Immunostaining of day 250

34 organoids revealed that $\sim 30 \%$ of SOX $2+\mathrm{NSCs}, \sim 60 \%$ of MAP $2+$ neurons, and $\sim 10 \%$ of GFAP+ astrocytes

35 were infected by WSN (Fig. 3K, L). The extracellular virus titers showed a time-dependent increase in WSN

36 infected day 250 organoids (Fig. 3M). These results suggested that MAP2+ neurons were more susceptible to

37 WSN infection compared to NSCs or astrocytes in brain organoids (Fig. 3N).

\section{Transcriptomic profiling of WSN infected organoids}

39 To investigate the underlying molecular mechanisms at the gene level, we performed transcriptomic profiling

40 of WSN-infected organoids at $1 \mathrm{dpi}$ and 4 dpi compared to mock-infected organoids. Hierarchical clustering 
41 analysis showed the significant differences in gene expression between 1 dpi and 4 dpi organoids compared to 42 mock-infected organoids (Fig. 4A). A Venn diagram shows that there were more gene alterations at 4 dpi compared to $1 \mathrm{dpi}$, and 266 genes were upregulated while 117 genes were downregulated in both groups (Fig.

4B). GO terms were mainly enriched in the regulation of cellular metabolic process, negative regulation of

developmental process, and innate immune response at $1 \mathrm{dpi}$, while they were mainly enriched in negative

regulation of nervous system development, and neuron differentiation at 4 dpi (Fig. 4C). KEGG signaling

pathway terms were enriched in virus infection pathways at $1 \mathrm{dpi}$, while they were enriched in MAPK

expression analysis at $1 \mathrm{dpi}$ and $4 \mathrm{dpi}$. In the top 10 of upregulated and downregulated genes, $F A B P 1, C D X 2$,

FGG, ISX, SI, GSTA1, GBP1P1, CXCL10, KRT20, and CXCL11 were significantly upregulated, while

MCIDAS, MFRP, CCKAR, CDC20B, AL355812.1, SLC39A12, CCNO, ABCA4, KCNJ13, and OTX2-AS1 were

significantly downregulated in the 1 dpi group. CCL7, HIST1H3PS1, RN7SL472P, NCOA4P2, WDR95P, IL6,

ZSCAN4, AP001331.1, AC108134.2, and GBP1P1 were significantly upregulated, while DAPL1, MIR217HG,

SIX6, AL451127.1, TFAP2D, AC016044.1,SIX3OS1_2,VSX1, CLRN1, and AL138826.1 were significantly

downregulated in the 4 dpi group (Fig. 4D). GBP1P1, CXCL10, CXCL11, CCL7, CSAG3, OAS2, and

NCOA4P2 were upregulated and $C D C 20 B, A L 355812.1, K C N J 13, O T X 2-A S 1, C R O C C 2$, and F5 were 
61 identified a set of ISGs (e.g., IFITM1/2/3, BST2, and SLC16A1) that were highly induced in the early stages of

62 WSN infection (1 dpi), while significantly decreased in late stages of infection (4 dpi) (Fig. 4F). This

63 indicated that ISGs played the crucial role against viral invasion during early infection. We also analyzed

64 some DEGs of transcription factors (Fig. S2B), inflammatory factors (Fig. S2C), and metabolic genes (Fig.

65 S2D) that were associated with virus infection. We found that they showed significant changes between 1 dpi

66 and 4 dpi compared to mock infection. Moreover, a protein-protein interaction network of WSN infected brain

67 organoids at 4 dpi was more robust than at 1 dpi (Fig. S2E). In addition, WSN-infected organoids caused not

68 only protein gene changes, but also some noncoding RNA levels, such as BISPR and MIR4435-2HG (Fig. S3).

69 Collectively, these results suggested that WSN infection of brain organoids resulted in considerable gene

70 alterations, and implicating these genes for the development of new antiviral strategies.

WNS impairs brain organoid growth through inducing apoptosis and inflammation

72 To further investigate the neuropathogenesis of brain organoids subjected to WSN infection, we conducted

73 related assays, including apoptosis and inflammatory factor release after WSN infection at $1 \mathrm{dpi}$ and $4 \mathrm{dpi}$ (Fig.

74 5A). Significant cell apoptosis of SOX2 + NSCs and MAP2+ neurons in day 30 and day 60 brain organoids at

751 dpi and 4 dpi was observed by TUNEL staining (Fig. 5B, C). The percentage of TUNEL+ cells was a

76 time-dependent increase, and the apoptosis of NSCs was higher than neurons during early WSN infection (Fig.

77 5D, E). Unlike Zika virus infection, which mainly induces the apoptosis of NSCs to result in

78 microcephaly(Tang et al., 2016), WSN primarily infects MAP2+ neurons and causes the apoptosis of both

79 NSCs and neurons. We also detected the apoptosis of GFAP+ astrocytes in day 250 organoids, and we did not

80 observe obvious apoptosis (Fig. 5F). Next, we determined the inflammatory factor levels in supernatants from 
81 days 30 and 60 brain organoids by enzyme linked immunosorbent assay (ELISA). We found that TNF- $\alpha$,

82 INF- $\gamma$, IL-6, CCL2, and COX2 in organoid supernatants were significantly increased in a time-dependent

83 manner from days 30 and 60 brain organoids (Fig. 5G, H). At the cellular level, we also confirmed that

84 influenza virus could induce NSC apoptosis (Fig. S1C, D) and inhibit proliferation (Fig. S1E, F), but did not

85 induce astrocyte apoptosis (Fig. S1I). Taken together, these results suggested that WSN could impair brain 86 organoid growth through eliciting apoptosis and inflammation (Fig. 5I).

87 Antiviral and neuroprotective effects of drugs and neurotrophic factors

88 As these results demonstrated, WSN did impair the human brain through inducing cell apoptosis and 89 inflammation responses using in vitro brain organoid models. Therefore, we performed antiviral screening 90 studies. We first analyzed several compounds, PYC-12, RO3306, and WHL-50B, at the cellular level, and we 91 used nucleozin as a positive control, as it induces nuclear accumulation of influenza virus nucleoprotein (NP) 92 leading to cessation of viral replication (Gerritz et al., 2011; Kao et al., 2010). Our results showed that 93 PYC-12, WHL-50B, and nucleozin all significantly suppressed virus infection compared to RO3306 (Fig. 94 S4A), and PYC-12 could also suppress WSN infection in astrocytes, but not as clearly as nucleozin (Fig. S4B). 95 Next, we further evaluated the antiviral effect of four compounds at the organoid level. Briefly, day 40 brain 96 organoids were first treated with four compounds for 2 hours, followed by co-treatment with WSN and 97 compounds for 1 hour, followed by continued compound treatment for the indicated number of days (Fig. 6A). 98 Representative bright field images showed that PYC-12 could significantly rescue the morphological changes 99 of brain organoids compared to the WSN infection group (Fig. 6B). Additionally, three compounds, peramivir, :00 PYC-12, and WHL-50B, were used in drug screening of H3N2-infected organoids, and the results showed that 
:01 peramivir as a positive drug, which is a highly selective inhibitor of influenza A and B neuraminidase (Kohno

:02 et al., 2011), had the highest antiviral effects compared to other drugs (Fig. S5). Statistical analysis of the

:03 diameter and area of organoids treated with PYC-12 also demonstrated its rescue effects (Fig. 6C, D).

:04 Therefore, we used PYC-12 for our following studies. Immunostaining of SOX2+ NSCs and MAP2+ neurons

:05 on day 30 and day 60 brain organoids infected by WSN revealed that PYC-12 treatment could significantly

:06 inhibit WSN infection (Fig. 6E) and cell apoptosis (Fig. 6F) at 1 dpi and 4 dpi. PYC-12 also decreased the

:07 extracellular and intracellular viral titers (Fig. 6G), as well as decreasing IL-6 and TNF- $\alpha$ production (Fig.

:08 6H). Microelectrode array (MEA) analysis showed that PYC-12 could increase the weighted mean firing rate

:09 (Hz) of brain organoids compared to WSN infection at indicated time points (Fig. 6I, J). Collectively, we

!10 screened a potential drug candidate-PYC-12 against WSN infection using brain organoid models (Fig. 6K).

:11 In addition, neurotrophic factors (NFs), which are endogenous soluble proteins regulating the survival and

:12 growth of neurons, protect human normal brain functions against microbial pathogens have been reported in

!13 the literature (Platholi and Lee, 2018). Thus, to investigate whether NFs also have antiviral effects in in vitro

:14 organoid models, we conducted antiviral assays with the same treatments (Fig. 6A). First, we demonstrated

:15 that brain-derived neurotrophic factor (BDNF), glial-derived neurotrophic factor (GDNF), neurotrophin-3

:16 (NT3), and their combinations (GBN) could significantly inhibit WSN infection at the NESTIN+ NSCs level

!17 (Fig. S6A), especially BDNF and GBN in NP+ cells (Fig. S6B). All three NFs and GBN showed decreased

!18 intracellular virus titers (Fig. S6C), while only GBN inhibited extracellular virus titers at the NSC level (Fig.

!19 S6D). Then, we confirmed the antiviral effect of GBN on day 30 organoids (Fig. S6E, F), and GBN showed

:20 decreases in intracellular and extracellular virus titers (Fig. S6G, H). GBN significantly inhibited WSN 
:21 infection through decreasing both cell apoptosis (Fig. S6I, J) and inflammatory factor release (Fig. S6K). At

:22 the gene level, we found that NF could significantly induce a set of ISG expressions, such as MCL1, IFITM3,

:23 B2M, BST2, OAS1, and PKR to function against virus infection (Fig. S7). Collectively, neurotrophic factors

:24 also possessed antiviral roles via inducing ISG expression to suppress cell apoptosis and inflammation

:25 response in brain organoid models (Fig. S6L), and implicating the possibility of a combination therapy in the :26 future. 


\section{:27 Discussion}

:28 The neuropathogenesis of the human brain caused by influenza virus remains poorly understood. In the light :29 of this fundamental problem, we designed an experimental platform using human brain organoids as a virus :30 infection model to study this neuropathogenesis in detail. Brain organoids serve as an invaluable tool in the :31 field of virus research to investigate the molecular events underlying virus replication and antiviral drug :32 screening.

:33 In this study, we first demonstrated that influenza virus (H1N1-WSN) could widely infect multiple cell types :34 in brain organoids, including SOX2+ neural stem cells (NSCs), MAP2+ neurons, and GFAP+ astrocytes, and :35 holds an infection tropism of MAP2+ neurons (Fig. 3), which may support a direct link between influenza :36 virus infection and the neurologic symptoms. Inflammatory factors (e.g., IL-6, TNF- $\alpha$, and INF- $\gamma$ ) induced by :37 WSN infection in brain organoids (Fig. 5G, H) may be the major cause for viral entrance into the brain :38 through damaging the blood brain barrier (BBB) (Shlosberg et al., 2010). However, whether other virulent :39 influenza viruses (e.g., H3N2, H5N1, or H7N9) have similar results needs further investigation in higher level :40 of biosafety laboratories. Unlike WSN infection, Zika virus preferentially infects human cortical neural :41 progenitor cells with high efficiency, but exhibits lower levels of infection in human embryonic stem cells :42 (ESCs), hPSCs, and immature cortical neurons (Wu et al., 2018). Therefore, regional and cell type specific :43 tropism of virus infection may directly support a link with specific pathological syndromes.

:44 As is well known, although brain organoids can serve as a model of a three-dimensional organ to achieve :45 multiple cellular interactions not possible with cell lines, organoids are very immature and only partly model :46 the human brain. Therefore, which type of neurons are specifically infected with influenza virus in brain 
:47 organoids needs further study in our next work. Some research groups have leveraged virulent viruses (e.g., :48 H5N1, H5N3, or H3N2) (Mori and Kimura, 2001) to infect mice, and have demonstrated the route of brain :49 infection, but cell type-specific tropism of viral infection in the central nervous system remain poorly :50 understood. Therefore, if we completely confirm the cell type-specific infections of influenza or other viruses, :51 it should provide a scientific basis for developing specific neurological disorder drugs. Single-cell RNA :52 sequencing technology (Hwang et al., 2018; Ofengeim et al., 2017) could be used to track specific cell types :53 infected by viruses in space and time through detecting the gene expression of viruses, while human brain :54 tissue infected by viruses are very hard to get and labeling hundreds or thousands of nerve cells is difficult for current single-cell RNA sequencing technology.

In addition, we found that WSN could infect astrocytes (Fig. 3J), but not induce their apoptosis (Fig. 5F, S1), which was similar to brain organoids infected with SARS-CoV-2 (Song et al., 2020). This finding may indicate that virus-infected astrocytes can lead to peripheral cell death by creating a locally hypoxic and a resource-restricted environment for cells. Furthermore, we performed whole RNA transcriptomic analysis for comparison of a control and 4 dpi and 1 dpi group and identified some upregulated protein targets (e.g., CCL7,

:61 NCOA4P2, GBP1P1, CXCL10, CXCL11, CSAG3, and OAS2) and noncoding RNAs (e.g., BISPR, 
:67 be validated in animal models. Next, we intend to conduct a high throughput antiviral drug screening study

:68 using this organoid platform. Moreover, we also explored the antiviral and neuroprotective effects of

:69 neurotrophic factors such as GDNF, BDNF, and NF3 (Fig. S6), and demonstrated that they also did suppress

:70 WSN infection, the apoptosis of NSCs and neurons, and inflammatory responses. This work also highlights

:71 the possibility of using a combinations therapy of drugs and neurotrophic factors in clinical treatment for viral

:72 infection.

\section{Conclusion}

:74 In this study, using an in vitro brain organoid model, we demonstrated that MAP2+ neurons were more :75 susceptible to influenza virus (H1N1-WSN) infection, unveiled the neuropathogenesis of the brain through :76 inducing apoptosis and inflammation, and conducted antiviral screening to achieve antiviral and

:77 neuroprotective aims. In summary, we establish a tractable experimental model system to investigate the :78 impact and mechanism of influenza virus on human brain development, and provide a platform for identifying :79 therapeutic compounds. 
Materials and Methods

\section{:87 Cell culture and reagents}

:88 Human HEK293T (CRL-11268) and Madin-Darby Canine Kidney (MDCK) cells (ATCC, CRL-2936) were

maintained in Dulbecco's Minimal Essential Medium (DMEM) (Gibco) supplemented with 10\% fetal bovine

serum (Gibco), 100 units/mL penicillin, and $100 \mu \mathrm{g} / \mathrm{mL}$ streptomycin (Invitrogen). Authentication and test for

:91 the free of mycoplasma were performed with MycAway ${ }^{\mathrm{TM}}$ one-step mycoplasma detection kit (Yeasen).

192 Astrocytes were purchased from Cellapy (CA2315106) and cultured in NeuroEasy maintenance medium

:93 (Cellapy). Human embryonic stem cells (hESCs) were obtained from Harvard Stem Cell Institute. hESCs were

194 routinely checked for pluripotent, normal karyotype, mycoplasma free and cultured in feeder-free conditions

295 on Matrigel-coated plates with Essential 8 medium (GIBCO) and passaged with TrypLE ${ }^{\mathrm{TM}}$ express (GIBCO).

:96 $10 \mu \mathrm{M}$ Peramivir (M3222, AbMole BioScience), $1 \mu \mathrm{M}$ Nucleozin (A3670, Apexbio), $50 \mu \mathrm{M}$ PYC-12, $20 \mu \mathrm{M}$

197 RO3306, $10 \mu \mathrm{M}$ WHL-50B, 20 ng/mL BDNF (450-02, PeproTech), 20 ng/mL GDNF (450-10, PeproTech),

:98 $20 \mathrm{ng} / \mathrm{mL}$ NT3 (450-03, PeproTech) were used in this study.

\section{Viruses}

:00 Three H1N1 strains (WSN, CA07 and PR8), Enterovirus 68/71, and SFTSV obtained from Academy of

;01 Military Medical Sciences were used in this study. All viruses' stocks were prepared in MDCK cells and

;02 titrated by TCID $_{50}$ on MDCK cells as described in details below. Studies with infectious H1N1 were

;03 conducted under biosafety level 2 (BSL-2) conditions at the Peking University Health Science Center with

;04 approval from Institutional Biosafety Committee.

;05 Neural stem cells (NSCs) differentiation 
hESCs were differentiated into NSCs on Matrigel-coated plates using the monolayer protocol as previously

;07 described(Lippmann et al., 2014). Briefly, hESCs were first dissociated into single cells with Accutase

;08 (STEMCELL). Then, hESCs were plated onto Matrigel at a density of $2 \times 10^{5}$ cells $/ \mathrm{cm}^{2}$ in E8 medium

containing $10 \mu \mathrm{M}$ ROCK inhibitor (STEMCELL Technologies) and cultured overnight. $24 \mathrm{~h}$ later, cells were

changed to E6 medium (GIBCO) containing $10 \mu \mathrm{M}$ SB431542 (STEMCELL) and 100 nM LDN193189

;11 (Selleckchem) to initiate differentiation. Medium was changed every day until day 7. Day 7-NSCs were used

;12 for the following experiments.

;13 Brain organoids generation and culture

;14 Brain organoids were generated from hESCs as previously described(Lancaster and Knoblich, 2014), but

;15 slightly modified. Briefly, for embryoid body (EB) formation, hESCs were washed twice with DPBS,

;16 incubated with Accutase for 5 minutes, and dissociated into single cells. 3000 single cells were seeded in each

;17 well of low attachment 96-well U-bottom plate in E8 medium containing $10 \mu \mathrm{M}$ ROCK inhibitor and

;18 centrifuged at $100 \mathrm{~g}$ for $3 \mathrm{~min}$, then medium was half changed every other day. On day 4, EBs were

;19 transferred to low attachment 24-well plate in neural induction medium containing DMEM-F12 (GIBCO) with

:20 1\% N2 supplement (GIBCO), 1\% Glutamax supplement (GIBCO), 1\% MEM-NEAA (GIBCO) and $1 \mu \mathrm{g} / \mathrm{mL}$

:21 Heparin (GIBCO), and medium was changed after 48 h. On day 7, EBs were transferred into Matrigel droplets

;22 as previously described and cultured in brain organoid differentiation media containing 50\% DMEM-F12, 50\%

;23 Neurobasal, 200x N2 supplement, 0.025\% Insulin (GIBCO), 100x Glutamax supplement, 200x MEM-NEAA,

i24 100x penicillin-streptomycin, 0.035\% 2-Mercaptoethanol and 100x B27 supplement without Vitamin A, and

;25 medium was changed after $48 \mathrm{~h}$. On day 10, organoids were transferred to orbital shaker (Corning) in brain 
;26 organoid differentiation media with Vitamin A, medium was changed every 4 days.

\section{;27 Virus infection}

;28 For cell line infection, H9, NSCs and astrocytes were seeded in chamber at $1 \times 10^{6}$ cells. The cells were then

:29 rinsed with PBS, and WSN was diluted to the desired multiplicity of infection (MOI) of 1 and added to the

i30 cells. The cells were incubated for $2 \mathrm{~h}$ at $37^{\circ} \mathrm{C}$. The supernatant was removed and the cells were washed twice

;31 with PBS. Culture medium with 1\% FBS and 1000x TPCK (final concentration $1 \mu \mathrm{g} / \mathrm{mL}$ ) was added to each

;32 well, and cells were incubated at $37{ }^{\circ} \mathrm{C}$ and $5 \% \mathrm{CO}_{2}$, after $24 \mathrm{~h}$ infection, cells were prepared for

i33 immunofluorescence staining. For brain organoids infection, organoids were transferred to low attachment

i34 24-well plate and washed twice with DPBS, and WSN was diluted to the desired multiplicity of infection

;35 (MOI) of 1 and added to the cultured medium. The organoids were incubated for $8 \mathrm{~h}$ at $37^{\circ} \mathrm{C}$. The supernatant

;36 was removed and the cells were washed twice with DPBS, and then organoids were transferred to low

;37 attachment 6-well plate, $4 \mathrm{~mL}$ culture medium with 1\% FBS and 1000x TPCK (final concentration $1 \mu \mathrm{g} / \mathrm{mL}$ )

;38 was added to each well, and organoids were incubated at $37{ }^{\circ} \mathrm{C}$ and $5 \% \mathrm{CO}_{2}$ at a shake speed of $60 \mathrm{rpm}$, after

;39 $24 \mathrm{~h}$ and $96 \mathrm{~h}$ infection, organoids were prepared for immunofluorescence staining, RNA extraction and

;40 RNA-seq, and the supernatant was harvested for ELISA and virus titration.

\section{;41 Immunofluorescence staining}

;42 For cell immunofluorescence staining, the cells were fixed with 4\% PFA at room temperature for 10 min,

i43 permeated with PBST (PBS with $0.1 \%$ Triton X-100) for 20 min and blocked with 1\% BSA for 30 min. Then

;44 the cells were incubated with primary antibodies listed in Supplementary Table 1 at $4{ }^{\circ} \mathrm{C}$ overnight. The i45 cells were subsequently incubated with secondary antibodies listed in Supplementary Table 1 at room 
;46 temperature for $1 \mathrm{~h}$. The cells were mounted with mounting fluid containing DAPI (Yeason, 36308ES11). For

;47 organoid immunofluorescence staining, the organoids were fixed with 4\% PFA at room temperature for 30

;48 min, then immersed in 30\% (w/v) sucrose until submersion before embedding and freezing in the Optimal

;49 Cutting Temperature (OCT) compound (Tissue-Tek). Serial $12 \mu \mathrm{m}$ sections were obtained by cryo-sectioning

;50 of the embedded organoid at $-20{ }^{\circ} \mathrm{C}$ using a cryostat (Leica). Cryosections were permeated with in PBST at

;51 temperature for $30 \mathrm{~min}$ and blocked with sheep serum (Zhongshanjinqiao, ZLI-9022) for $1 \mathrm{~h}$. The sections

;52 were incubated with primary antibodies listed in Supplementary Table 1 diluted in blocking buffer at $4{ }^{\circ} \mathrm{C}$

;53 overnight. The slides were subsequently incubated with secondary antibody at room temperature for $1 \mathrm{~h}$. The

;54 slides were mounted with mounting fluid containing DAPI. Stained sections were photographed under a

;55 Nikon Ti-S microscope. Apoptotic cells were labelled with Click-It Plus TUNEL assay (C10619,

;56 ThermoFisher Scientific).

;57 RNA isolation and quantitative RT-PCR

i58 Total RNA was extracted from cells or organoids using the Quick-RNA MicroPrep kit (Zymo Research).

;59 RNA was subjected to quantitative real-time PCR in accordance with the protocol provided by one-step SYBR

;60 green RT-PCR Kit (Cwbio). The transcripts were quantitated and normalized to the internal GAPDH control.

;61 The primers used in the experiments are listed in Supplementary Table 2. The PCR conditions were 1 cycle

;62 at $95{ }^{\circ} \mathrm{C}$ for $5 \mathrm{~min}$, followed by 40 cycles at $95{ }^{\circ} \mathrm{C}$ for $15 \mathrm{~s}, 60^{\circ} \mathrm{C}$ for $1 \mathrm{~min}$, and 1 cycle at $95{ }^{\circ} \mathrm{C}$ for $15 \mathrm{~s}$,

;63 $60{ }^{\circ} \mathrm{C}$ for $15 \mathrm{~s}, 95{ }^{\circ} \mathrm{C}$ for $15 \mathrm{~s}$. The results were calculated using the $2^{-\Delta \Delta \mathrm{CT}}$ method according to the GoTaq

;64 qPCR Master Mix (Promega) manufacturer's specifications.

;65 Virus quantification by $\mathbf{5 0 \%}$ tissue culture infective dose 
;66 For quantifying all viruses' stocks, the 50\% tissue culture infectious dose (TCID $50 / \mathrm{mL})$ titers were determined.

;67 In brief, $5 \times 10^{4}$ HEK293T cells were seeded in 96-well plates the day before infection. The virus samples were ;68 serially diluted with DMEM containing $1 \%$ FBS $\left(10^{3}\right.$ to $\left.10^{10}\right)$ and then each of dilution was added in wells ;69 separately. The plates were incubated at $37{ }^{\circ} \mathrm{C}$ in $5 \% \mathrm{CO}_{2}$ for $2-5$ days. The cytopathic effect (CPE) was ;70 observed under a microscope and determined virus titer using the Reed-Münch endpoint calculation method.

\section{;71 Microelectrode arrays (MEA)}

;72 Day 40 brain organoids were seeded onto 48-well transparent MEA plates. Brain organoids were cultured in ;73 brain organoid differentiation media containing 50\% DMEM-F12, 50\% Neurobasal, 200x N2 supplement, i74 0.025\% Insulin (GIBCO), 100x Glutamax supplement, 200x MEM-NEAA, 100x penicillin-streptomycin, ;75 0.035\% 2-Mercaptoethanol and 100x B27 supplement with Retinoic Acid (RA). MEA recordings were ;76 performed on day 3, 5, 7, 9, 11, 13 at $37{ }^{\circ} \mathrm{C}$ in a Maestro MEA system with AxIS software using a bandwidth ;77 with a filter for $10 \mathrm{~Hz}$ to $2.5 \mathrm{kHz}$ cutoff frequencies. For the pharmacological experiment, $50 \mu \mathrm{M}$ PYC-12 were applied to plate immediately before recording. For MEA recording, brain organoids treated with WSN were

;79 included as the control organoids. The phase contrast images of organoids seeded in the MEA plates were ;80 taken after MEA recording.

\section{;81 Enzyme-linked immunosorbent assay (ELISA)}

;82 Inflammatory factors (TNF- $\alpha$, INF- $\gamma$, IL-6, CCL2, COX2) in cultured supernatants of brain organoids with or ;83 without challenging by virus was measured using a commercial ELISA Kit (Dogesce). Briefly, samples were ;84 double diluted using the dilution buffer, and the optical density (OD) was measured at 450 nm with an ELISA ;85 reader (Beckman). The concentration of inflammatory factors was calculated according to the manufacturer's 
instruction.

\section{;87 The whole transcriptome analysis}

;88 High throughput RNA sequencing was performed by Cloud-Seq Biotech (Shanghai, China). Total RNA was

extracted from organoids (three biological replicates for each group) by TRIzol and the rRNAs were removed

with NEBNext rRNA Depletion Kit (New England Biolabs, Inc., Massachusetts, USA). RNA libraries were

constructed using the NEBNext巴 Ultra ${ }^{\mathrm{TM}}$ II Directional RNA Library Prep Kit (New England Biolabs, Inc.,

Massachusetts, USA) following the manufacturer's instructions. The libraries were quality-controlled and

performed on an Illumina Hiseq instrument with 150 bp paired end reads. Paired-end reads were harvested

from Illumina HiSeq 4000 sequencer, and quality-controlled by Q30. After 3' adaptor-trimming and removing

low-quality reads by cutadapt software (v1.9.3), high-quality clean reads were aligned to the reference genome

(UCSC MM10) with hisat2 software (v2.0.4). Guided by the Ensembl gtf gene annotation file, the cuffdiff

software (part of cufflinks) was used to obtain the gene level FPKM as the expression profiles of mRNA. The

total expressed gene number and LogFPKM of mRNA in different mouse groups were plotted and compared.

\section{Statistical analysis}

101 All data were analyzed using the GraphPad Prism 9 software. For the statistical analysis of other results,

102 statistical evaluation was performed by Student's unpaired t-test or one-way ANOVA with Tukey's multiple

103 comparisons test. Data are presented as means $\pm \mathrm{SD}$ or as described in the corresponding legends. A

t04 probability of $\mathrm{p}<0.05$ was considered as statistically significant. For annotations of significance, ${ }^{*} \mathrm{p}<0.05$;

$$
{ }^{* *} \mathrm{p}<0.01 ;{ }^{* * *} \mathrm{p}<0.001 ;{ }^{* * * *} \mathrm{p}<0.0001
$$




\section{Data availability}

107 All other data supporting this study are available within this paper and its Supplementary Information. 108 


\section{H10 Acknowledgements}

111 This work was funded by China's National Science and Technology Major Projects for Major New Drugs

H12 Innovation and Development (No. 2018ZX09711003-001-003).

\section{H13 Author contributions}

H14 Q. X. conceived the idea and X. Z. initiated the project. X. Z. and H. L. analyzed the sequencing data, H15 prepared the figures, and wrote the draft. Z. X. performed the flow cytometry and cytokine release activity H16 assays. All authors had access to the data. K. Y., L. T. and L. D. verified the data. All co-first authors and H17 corresponding authors discussed the results and revised the paper.

\section{H18 Additional information}

H19 Supporting information is available for this paper.

\section{Conflict of interest statement}

121 The authors have declared that no conflict of interest exists.

122 


\section{References}

124 Cao, J., Lu, G., Wen, L., Luo, P., Huang, Y., Liang, R., Tang, K., Qin, Z., Chan, C.C.Y., Chik, K.K.H., et al. 125 (2021). Severe fever with thrombocytopenia syndrome virus (SFTSV)-host interactome screen identifies viral 126 nucleoprotein-associated host factors as potential antiviral targets. Comput. Struct. Biotechnol. J. 19, $127 \quad 5568-5577$.

128 Chen, X., Sun, G., Tian, E., Zhang, M., Davtyan, H., Beach, T.G., Reiman, E.M., Blurton-Jones, M., 129 Holtzman, D.M., and Shi, Y. (2021). Modeling Sporadic Alzheimer's Disease in Human Brain Organoids under Serum Exposure. Adv. Sci. 8, 1-16.

De Crignis, E., Hossain, T., Romal, S., Carofiglio, F., Moulos, P., Khalid, M.M., Rao, S., Bazrafshan, A.,

Verstegen, M.M.A., Pourfarzad, F., et al. (2021). Application of human liver organoids as a patient-derived primary model for HBV infection and related hepatocellular carcinoma. Elife 10, 1-32.

Das, K., Aramini, J.M., Ma, L.C., Krug, R.M., and Arnold, E. (2010). Structures of influenza A proteins and insights into antiviral drug targets. Nat. Struct. Mol. Biol. 17, 530-538.

141 Gerritz, S.W., Cianci, C., Kim, S., Pearce, B.C., Deminie, C., Discotto, L., McAuliffe, B., Minassian, B.F., Shi, 142 S., Zhu, S., et al. (2011). Inhibition of influenza virus replication via small molecules that induce the formation 
143 of higher-order nucleoprotein oligomers. Proc. Natl. Acad. Sci. U. S. A. 108, 15366-15371.

144 Han, J., Perez, J., Schafer, A., Cheng, H., Peet, N., Rong, L., and Manicassamy, B. (2018). Influenza Virus:

145 Small Molecule Therapeutics and Mechanisms of Antiviral Resistance. Curr. Med. Chem. 25, 5115-5127.

146 Hoekstra, P.J. (2019). Attention-deficit/hyperactivity disorder: is there a connection with the immune system?

147 Eur. Child Adolesc. Psychiatry 28, 601-602.

148 Hwang, B., Lee, J.H., and Bang, D. (2018). Single-cell RNA sequencing technologies and bioinformatics 149 pipelines. Exp. Mol. Med. 50, 1-14.

150 Johansson, A., Mohamed, M.S., Moulin, T.C., and Schïoth, H.B. (2020). Neurological manifestations of

151 COVID-19: A comprehensive literature review and discussion of mechanisms. J. Neuroimmunol. 358, 152 577658.

153 Kao, R.Y., Yang, D., Lau, L.S., Tsui, W.H.W., Hu, L., Dai, J., Chan, M.P., Chan, C.M., Wang, P., Zheng, B.J., I54 et al. (2010). Identification of influenza A nucleoprotein as an antiviral target. Nat. Biotechnol. 28, 600-605.

155 Keipp Talbot, H., and Falsey, A.R. (2010). The diagnosis of viral respiratory disease in older adults. Clin. 156 Infect. Dis. 50, 747-751.

157 Kohno, S., Kida, H., Mizuguchi, M., Hirotsu, N., Ishida, T., Kadota, J., Shimada, J., and Seki, M. (2011). 158 Intravenous peramivir for treatment of influenza A and B virus infection in high-risk patients. Antimicrob. 159 Agents Chemother. 55, 2803-2812.

t60 Koyuncu, O.O., Hogue, I.B., and Enquist, L.W. (2013). Virus infections in the nervous system. Cell Host I61 Microbe 13, 379-393.

t62 Lancaster, M.A., and Knoblich, J.A. (2014). Generation of cerebral organoids from human pluripotent stem 
I63 cells. Nat. Protoc. 9, 2329-2340.

164 Liang, C.Y., Yang, C.H., and Lin, J.N. (2018). Focal Encephalitis, Meningitis, and Acute Respiratory Distress

65 Syndrome Associated with Influenza A Infection. Med. Princ. Pract. 27, 193-196.

166 Lippmann, E.S., Estevez-Silva, M.C., and Ashton, R.S. (2014). Defined human pluripotent stem cell culture 167 enables highly efficient neuroepithelium derivation without small molecule inhibitors. Stem Cells 32, 168 1032-1042.

I69 Malterer, M.B., Glass, S.J., and Newman, J.P. (2014). Interferon-stimulated genes: A complex web of host 170 defenses. Annu. Rev. Immunol. 44, 735-745.

171 McGavern, D.B., and Kang, S.S. (2011). Illuminating viral infections in the nervous system. Nat. Rev. 172 Immunol. 11, 318-329.

173 Mori, I., and Kimura, Y. (2001). Neuropathogenesis of influenza virus infection in mice. Microbes Infect. 3, $174 \quad 475-479$.

175 Ofengeim, D., Giagtzoglou, N., Huh, D., Zou, C., and Yuan, J. (2017). Single-Cell RNA Sequencing: 176 Unraveling the Brain One Cell at a Time. Trends Mol. Med. 23, 563-576.

177 Ooi, M.H., Wong, S.C., Lewthwaite, P., Cardosa, M.J., and Solomon, T. (2010). Clinical features, diagnosis, 178 and management of enterovirus 71. Lancet Neurol. 9, 1097-1105.

179 Platholi, J., and Lee, F.S. (2018). Chapter 5 - Neurotrophic Factors (Elsevier Inc.).

180 Qian, X., Song, H., and Ming, G. (2019). Brain organoids: advances, applications and challenges. 181 Development 146, dev166074.

182 Sachs, N., de Ligt, J., Kopper, O., Gogola, E., Bounova, G., Weeber, F., Balgobind, A.V., Wind, K., Gracanin, 
183 A., Begthel, H., et al. (2018). A Living Biobank of Breast Cancer Organoids Captures Disease Heterogeneity.

184 Cell 172, 373-386.e10.

185 Sauer, A.K., Stanton, J.E., Hans, S., and Grabrucker, A.M. (2021). Autism Spectrum Disorders: Etiology and

186 Pathology. Autism Spectr. Disord. Chapter 1, 1-16.

187 Shlosberg, D., Benifla, M., Kaufer, D., and Friedman, A. (2010). Blood-brain barrier breakdown as a 188 therapeutic target in traumatic brain injury. Nat. Rev. Neurol. 6, 393-403.

189 Song, E., Zhang, C., Israelow, B., Lu, P., Weizman, O., Liu, F., Dai, Y., Szigeti-buck, K., Yasumoto, Y.,

190 Wang, G., et al. (2020). Neuroinvasive potential of SARS-CoV-2 revealed in a human brain organoid model

191 Eric. BioRxiv.

192 Tang, H., Hammack, C., Ogden, S.C., Wen, Z., Qian, X., Li, Y., Yao, B., Shin, J., Zhang, F., Lee, E.M., et al.

193 (2016). Zika virus infects human cortical neural progenitors and attenuates their growth. Cell Stem Cell 18,

$194 \quad 587-590$.

195 Tregoning, J.S., and Schwarze, J. (2010). Respiratory viral infections in infants: Causes, clinical symptoms, 196 virology, and immunology. Clin. Microbiol. Rev. 23, 74-98.

197 Vehapoglu, A., Turel, O., Uygur Sahin, T., Kutlu, N.O., and Iscan, A. (2015). Clinical Significance of Human 198 Metapneumovirus in Refractory Status Epilepticus and Encephalitis: Case Report and Review of the Literature.

199 Case Rep. Neurol. Med. 2015, 1-4.

;00 Van De Wetering, M., Francies, H.E., Francis, J.M., Bounova, G., Iorio, F., Pronk, A., Van Houdt, W., Van ;01 Gorp, J., Taylor-Weiner, A., Kester, L., et al. (2015). Prospective derivation of a living organoid biobank of ;02 colorectal cancer patients. Cell 161, 933-945. 
;03 Wu, X., Dao Thi, V.L., Huang, Y., Billerbeck, E., Saha, D., Hoffmann, H.H., Wang, Y., Silva, L.A.V., ;04 Sarbanes, S., Sun, T., et al. (2018). Intrinsic Immunity Shapes Viral Resistance of Stem Cells. Cell 172, ;05 423-438.e25.

;06 Yu, J. (2021). Organoids: A New Model for SARS-CoV-2 Translational Research. Int. J. Stem Cells 14, ;07 138-149. 


\section{¡10 Figures and legends}

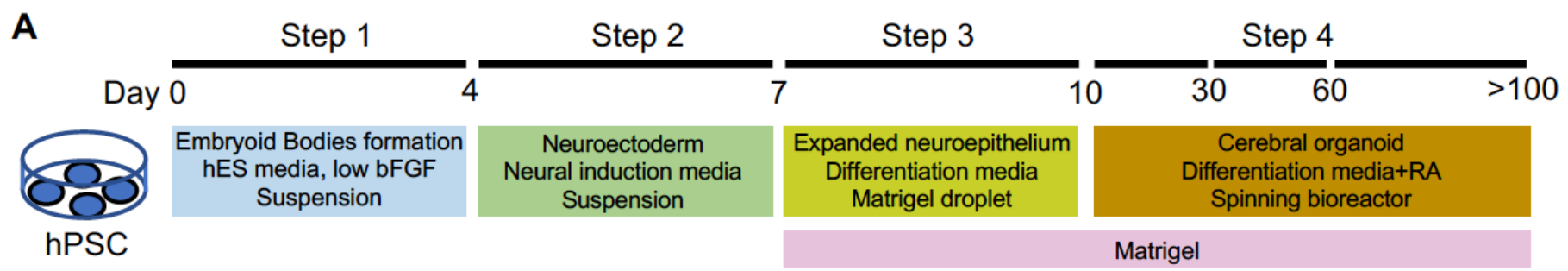

B
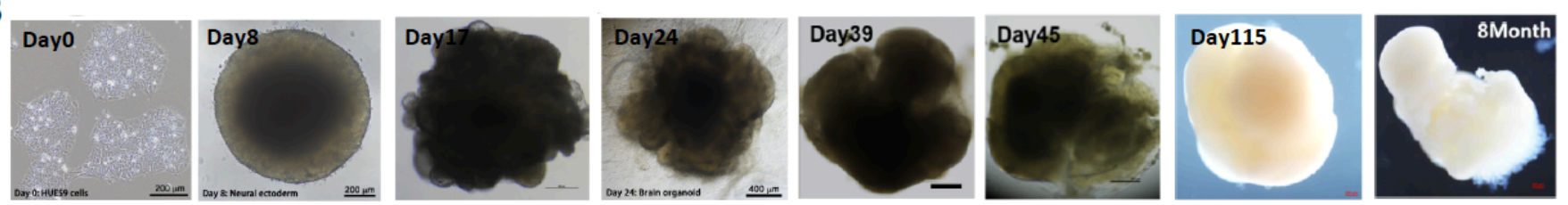

C

D
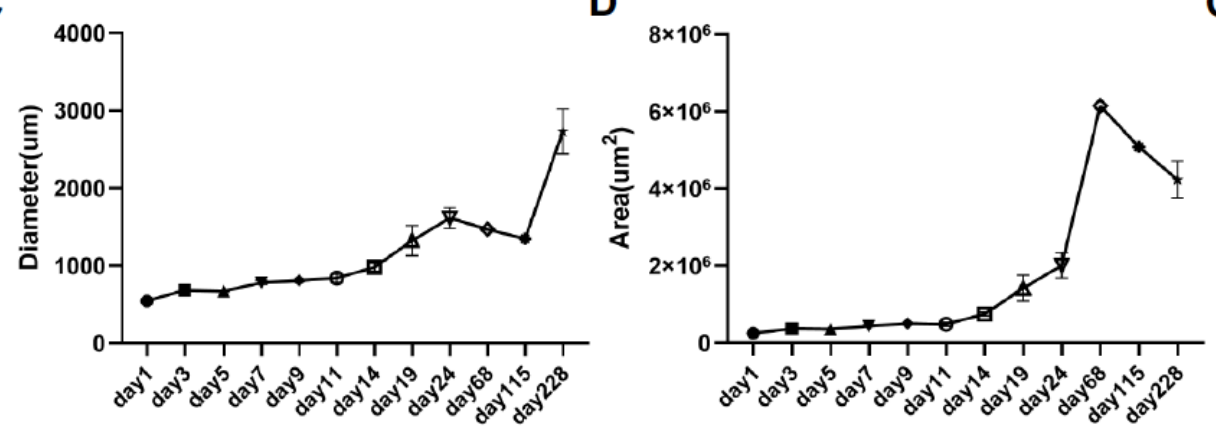

G

$\mathbf{F}$

E
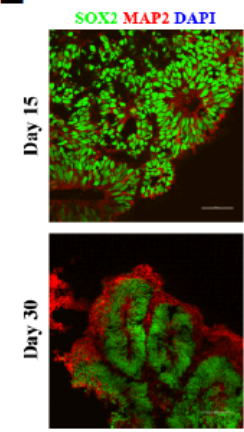

H

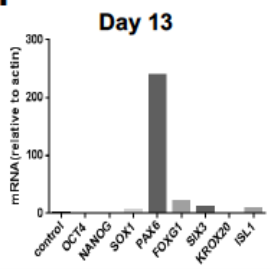

DAPI
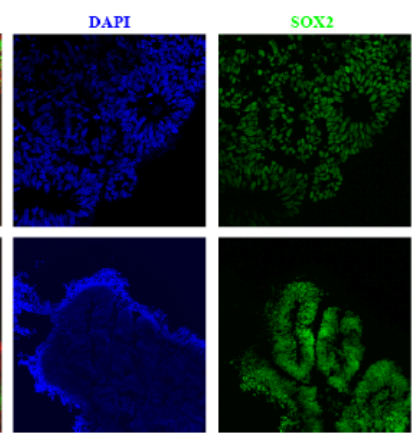
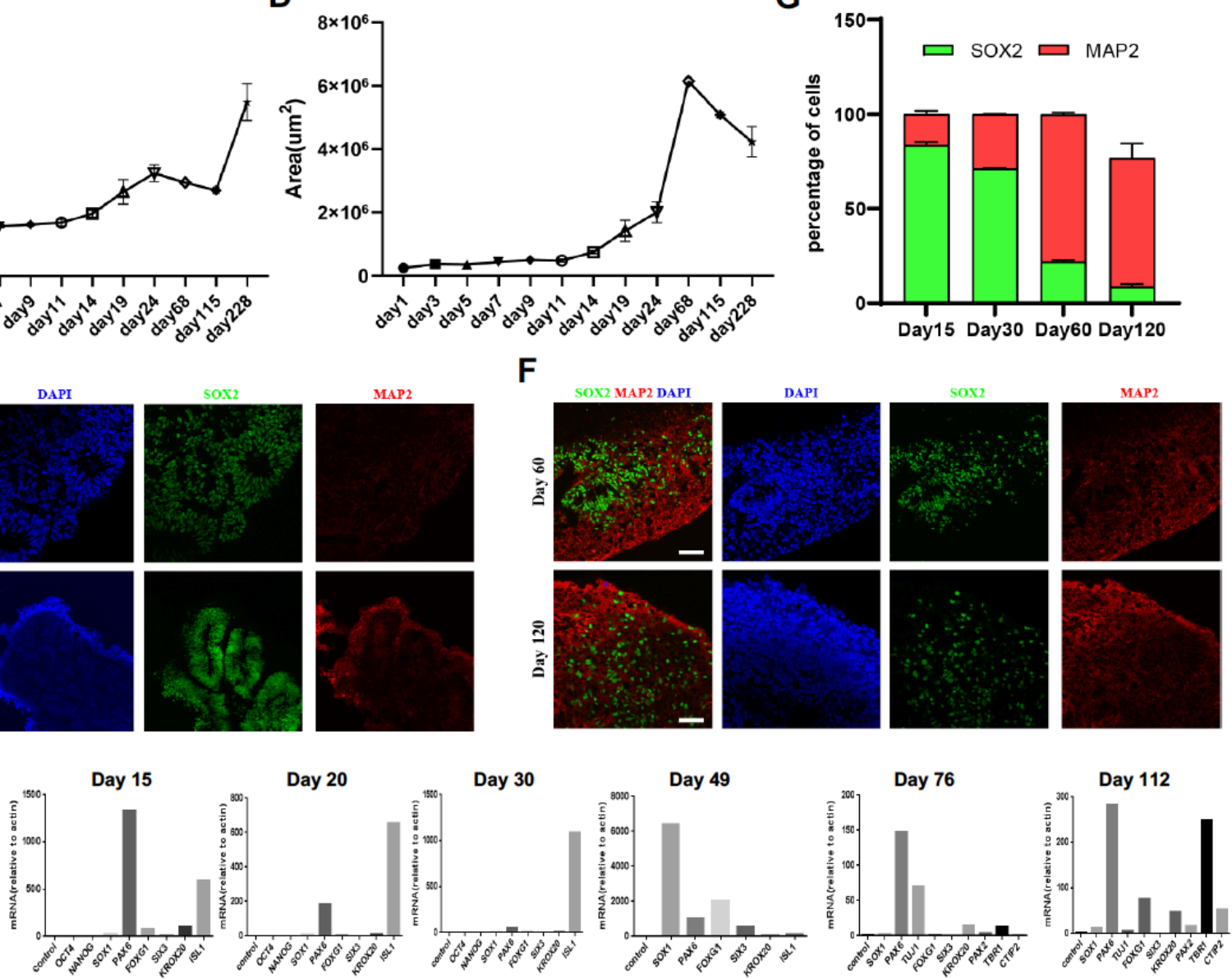

;12 Figure 1. Generation and characterization of brain organoids. (A) Schematic illustration of organoid

i13 generation. (B) Representative bright-field images of brain organoids derived from hPSCs at day 0, day 8, day 
;14 17, day 24, day 39, day 45, day 115, and 8 months. Scale bars, $200 \mu \mathrm{m}$ and $400 \mu \mathrm{m}$. (C, D) Growth kinetics of ;15 area $\left(\mu \mathrm{m}^{2}\right)$ and diameter $(\mu \mathrm{m})$ of brain organoids during development. (E, F) Immunostaining of brain ;16 organoids with SOX2+ NSCs and MAP2+ neurons on day 15, 30, 60 and 120. Scale bars, $100 \mu \mathrm{m}$. (G) The ;17 percentage of SOX2+ and MAP2 + cells in day 15, 30, 60, and 120 brain organoids. The majority of cells on ;18 days 15 and 30 organoids were SOX2+ neural stem cells and MAP2+ neurons on days 60 and 120 organoids. ;19 (H) Quantitative RT-PCR analysis of the specific markers of brain organoids at indicated timepoints. hPSC ;20 markers: OCT4 and NANOG; NSC marker: PAX6; forebrain markers: FOXG1 and SIX3; hindbrain markers: ;21 KROX20 and ISL1; neuron marker: TUJ1; deep-layer cortical neuron markers: CTIP2 and TBR1. $; 22$ 
A

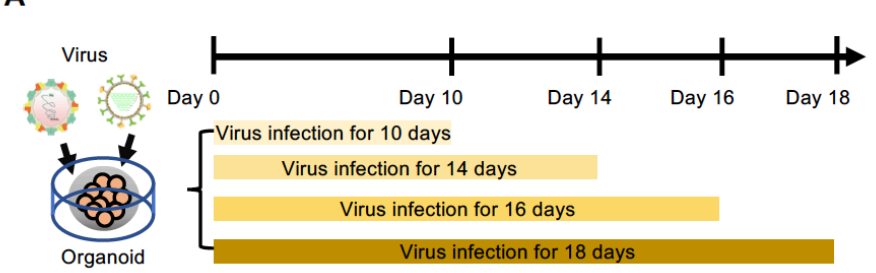

B

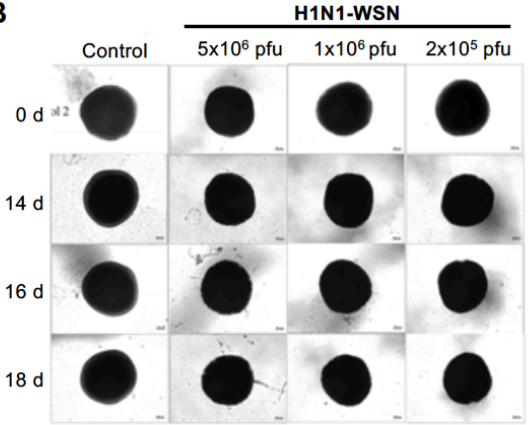

D

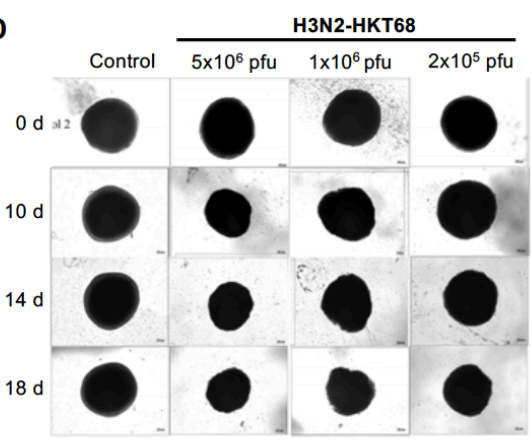

$C_{2200}=$ wSN-5x106
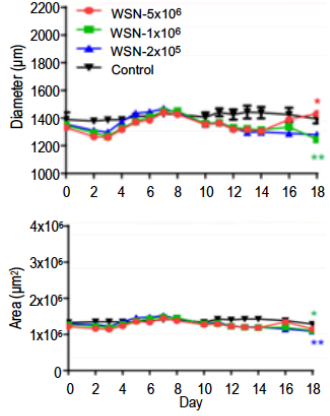

E

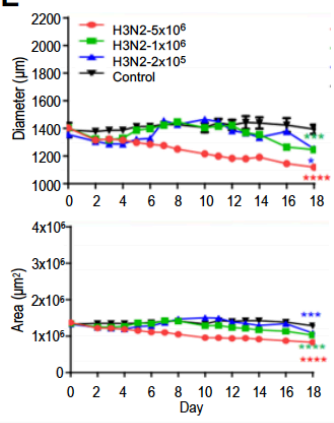

$\mathbf{F}$

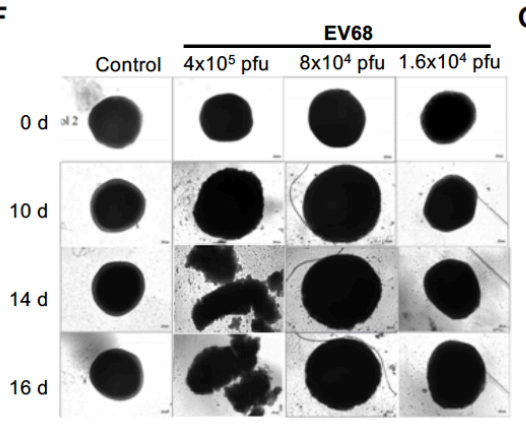

H
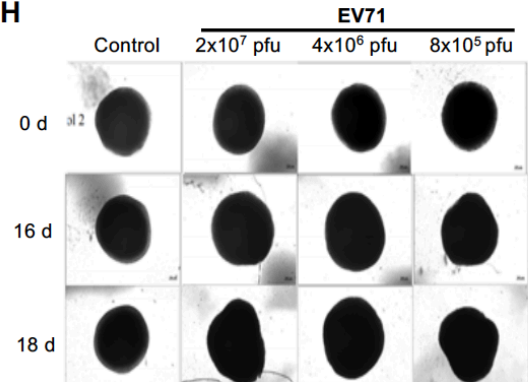

$\mathbf{J}$

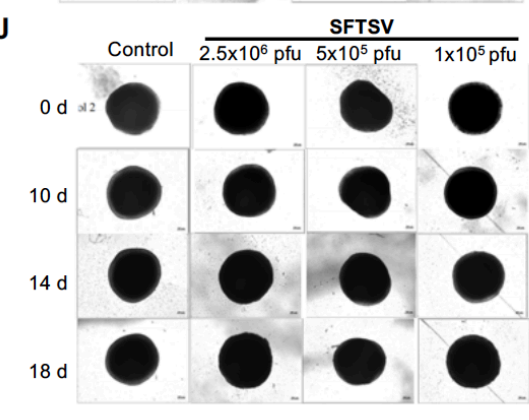

G
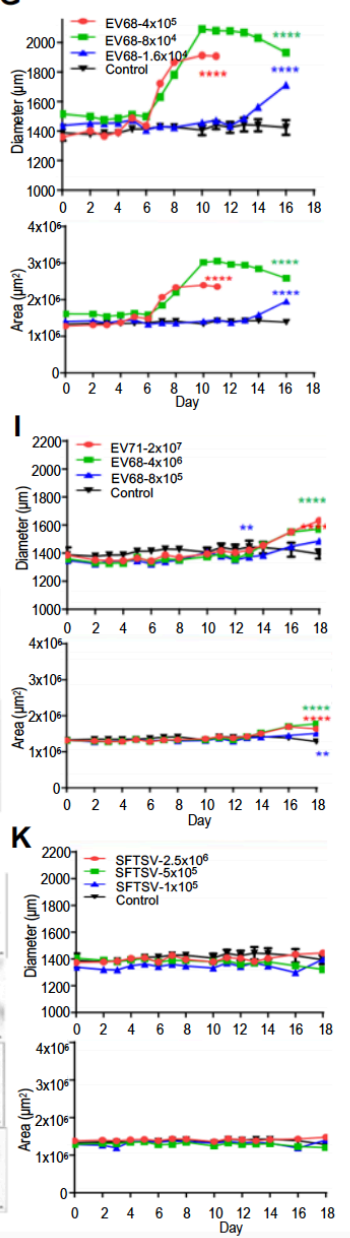

Figure 2. Organoids as virus infection models. (A) Schematic illustration of experimental design. Day 40

brain organoids were infected with viruses at indicated times. (B, D, F, H, J) Representative bright-field images of day 40-brain organoids infected with viruses at indicated time points, including H1N1-WSN, pfu, and $2 \times 10^{5} \mathrm{pfu}$; for H3N2, they were $5 \times 10^{6} \mathrm{pfu}, 1 \times 10^{6} \mathrm{pfu}$, and $2 \times 10^{6} \mathrm{pfu}$; for EV 68 , they were $4 \times 10^{5} \mathrm{pfu}$, $8 \times 10^{4} \mathrm{pfu}$, and $1.6 \times 10^{4} \mathrm{pfu}$; for EV71, they were $2 \times 10^{7} \mathrm{pfu}, 4 \times 10^{6} \mathrm{pfu}$, and $8 \times 10^{5} \mathrm{pfu}$. Scale bars, $50 \mu \mathrm{m}$. (C, indicated time points. 


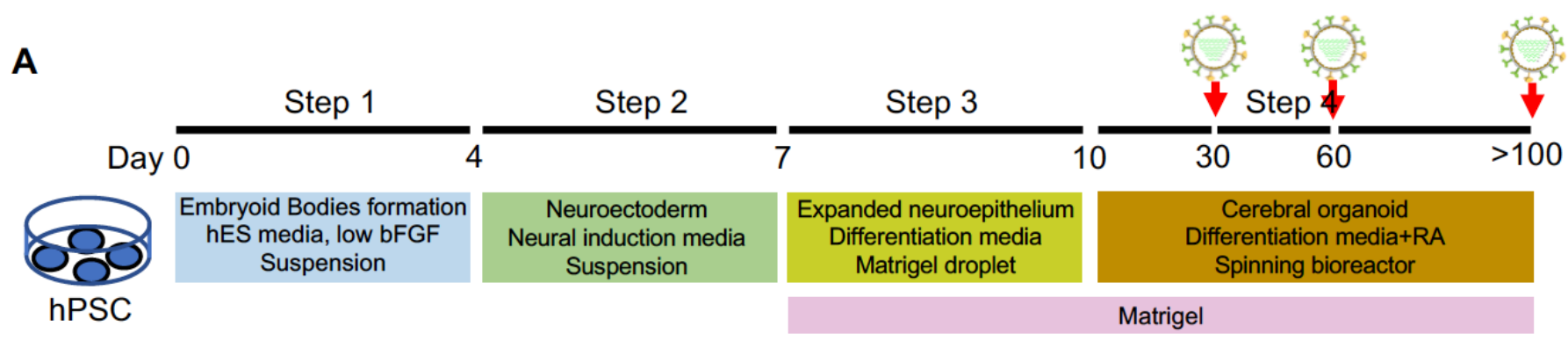

B

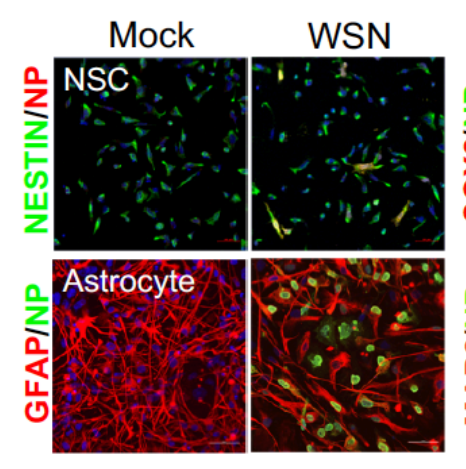

C

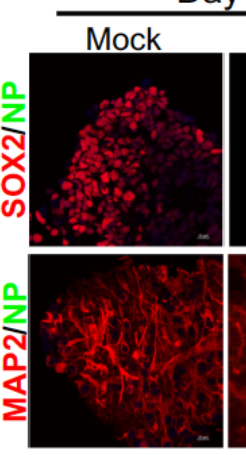

Day 30-organoid

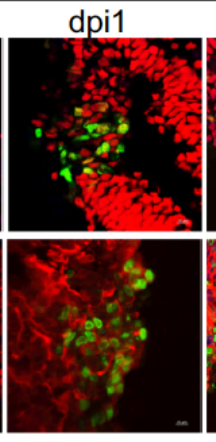

D

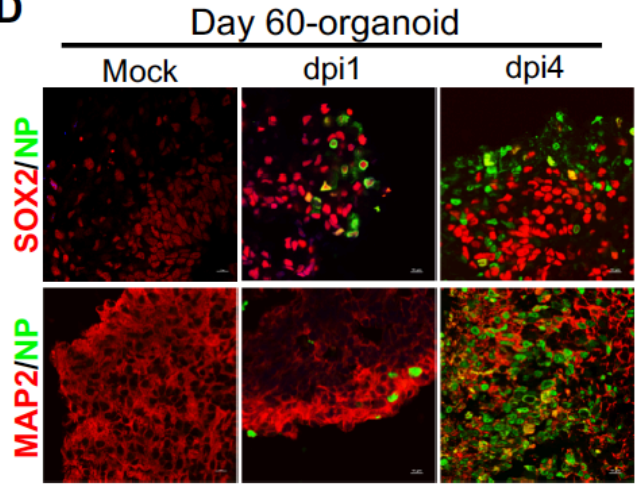

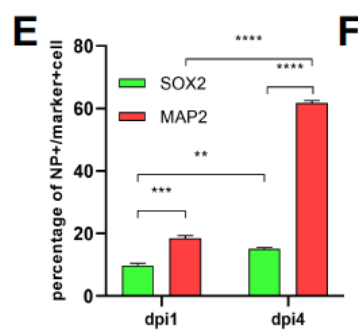
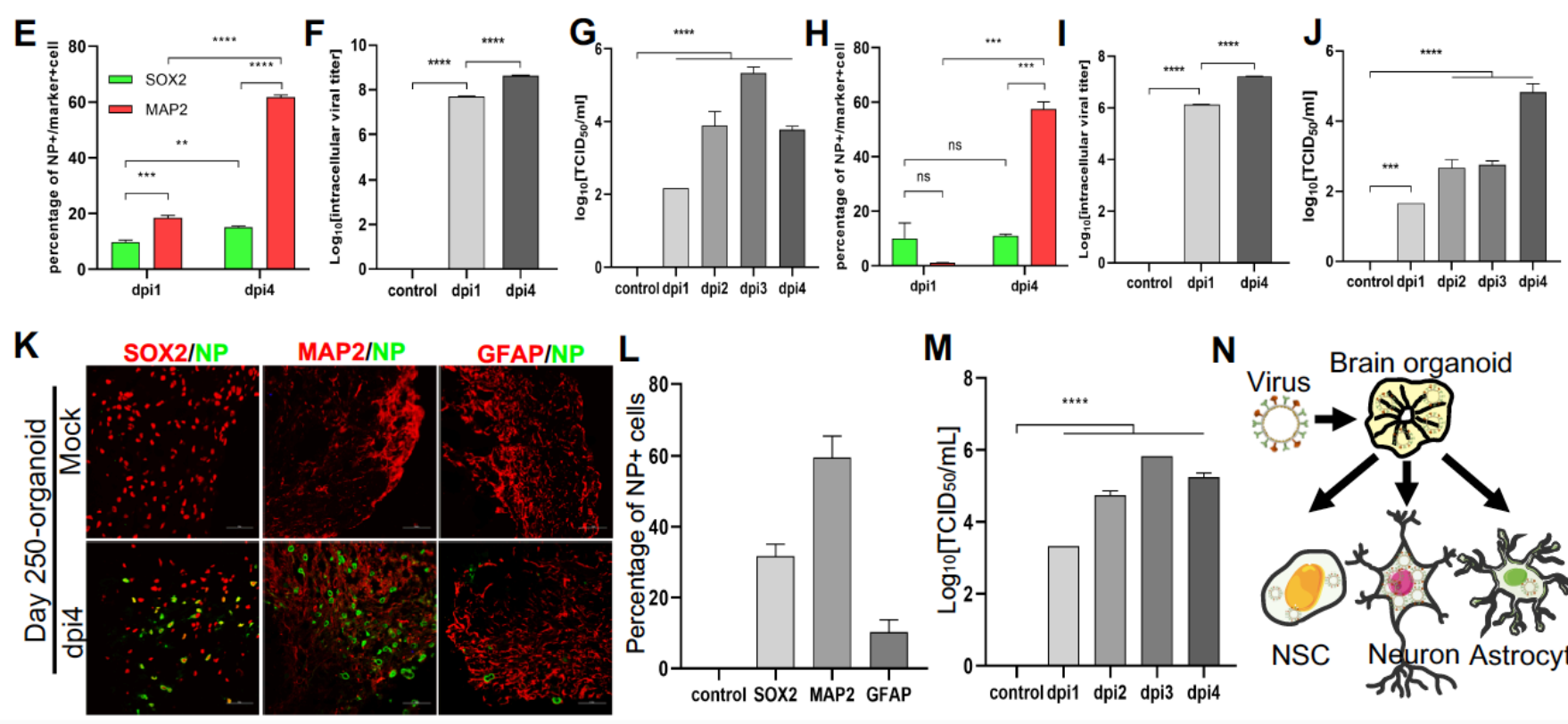

Brain organoid

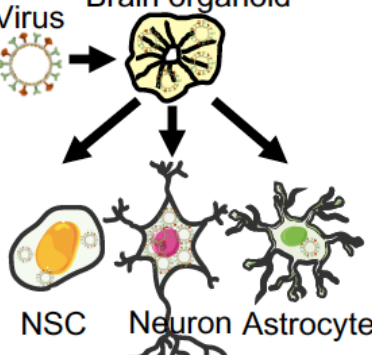

siti

¡34 Figure 3. Modeling influenza virus infection in vitro using brain organoids. (A) Schematic illustration of

i35 the experimental design of brain organoids infected with WSN at indicated time points. (B) Immunostaining

;36 of neural stem cells and astrocytes infected with WSN. Scale bars, $50 \mu \mathrm{m}$. (C, D) Immunostaining of neural

;37 stem cells and neurons in day 30 and day 60 brain organoids infected with WSN for 1 day and 4 days. Scale 
;38 bars, $100 \mu \mathrm{m}$. (E, H) The percentage of NP+ cells in infected day 30 and day 60 brain organoids. $\sim 10 \%$ of ;39 SOX2+ NSCs and $\sim 60 \%$ of MAP2+ neurons were infected with WSN. (F, G, I, J) The viral titers of ;40 intracellular and supernatants in day 30 and day 60 brain organoids. (K) Immunostaining of neural stem cells, ;41 neurons and astrocytes of day 250 brain organoids infected with WSN for 1 day and 4 days. Scale bars, 100 ;42 $\mu \mathrm{m}$. (L) The percentage of NP+ cells in infected brain organoids. $\sim 30 \%$ of SOX2+ NSCs, $\sim 60 \%$ of MAP2+ ;43 neurons and 10\% astrocytes were infected with WSN. (M) The viral titers of supernatants on day 250 brain ;44 organoids. (N) Schematic illustration of WSN preferentially infected neurons in brain organoids. ¡45 
A

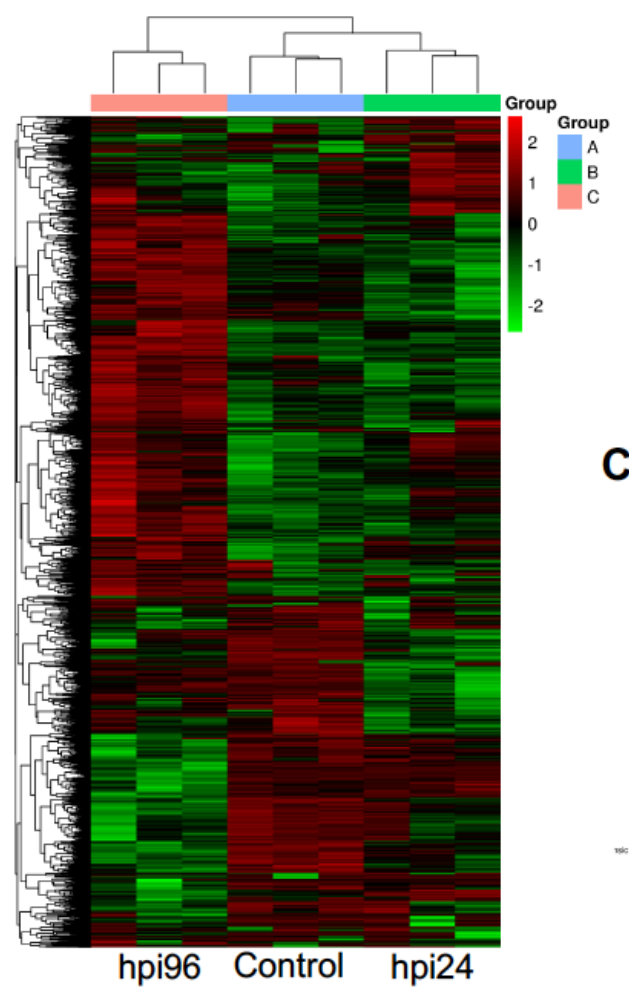

C

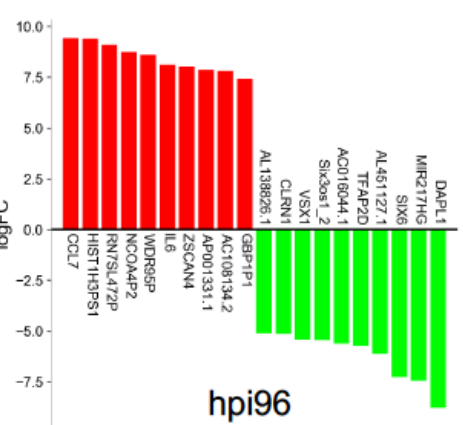

E
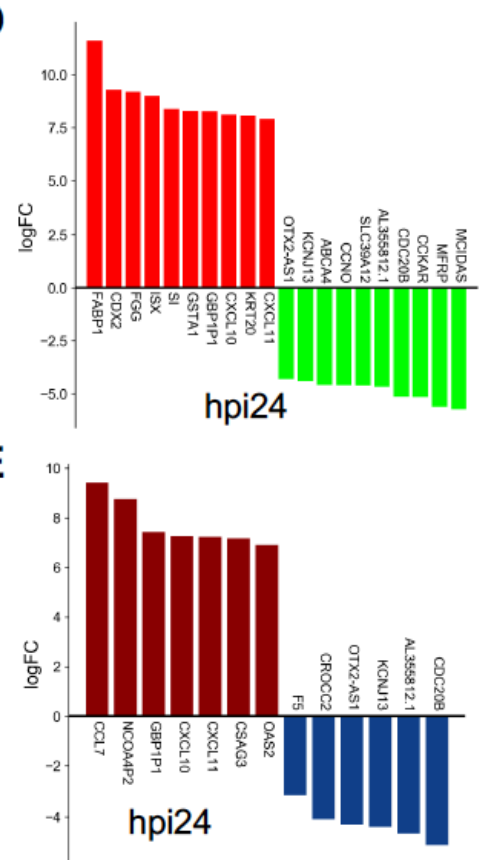

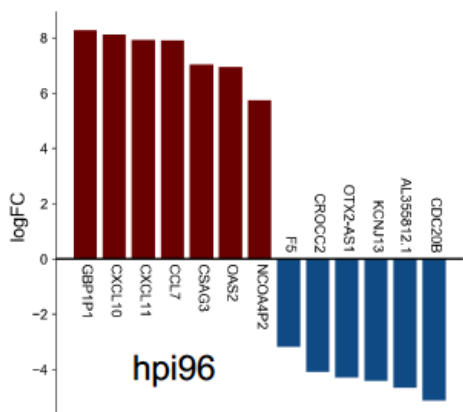

B

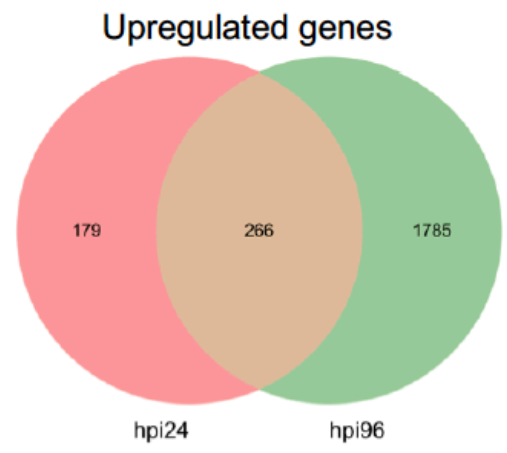

Downregulated genes
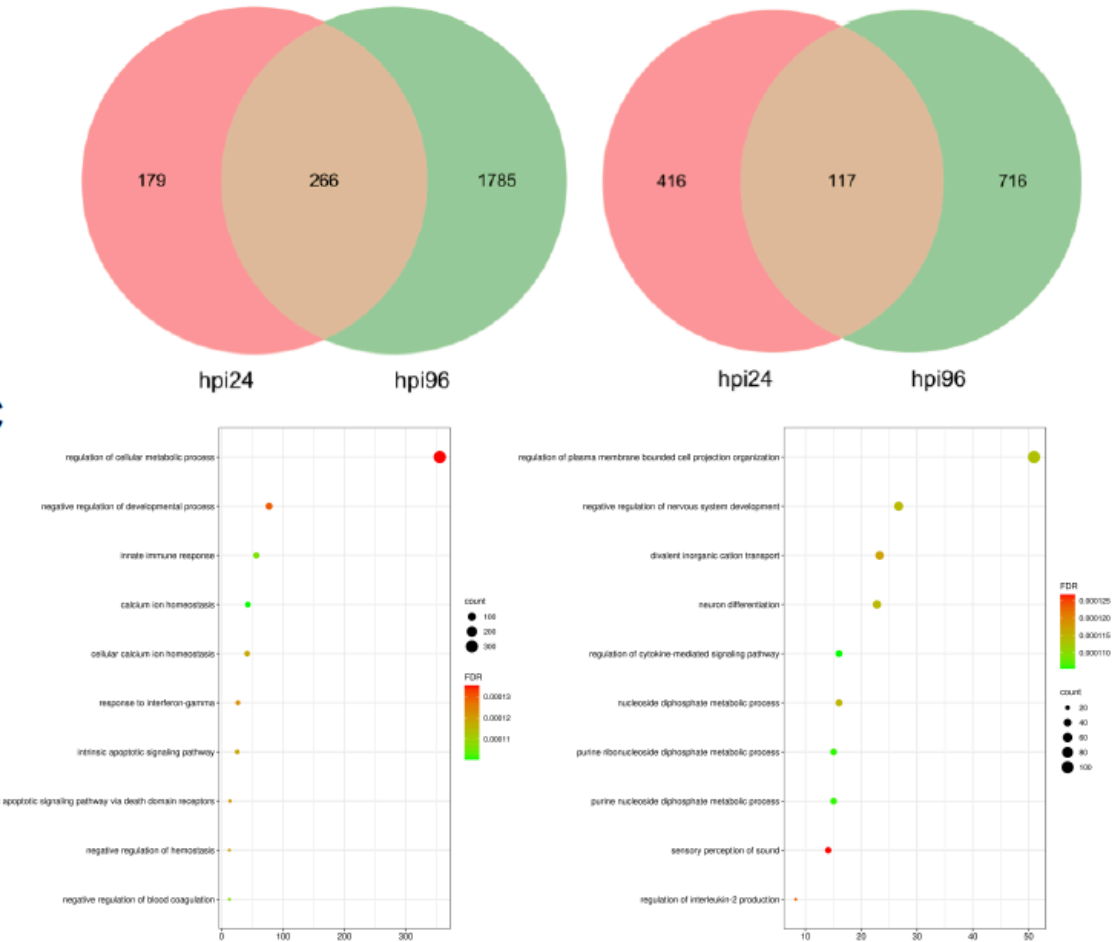

$\mathbf{F}$

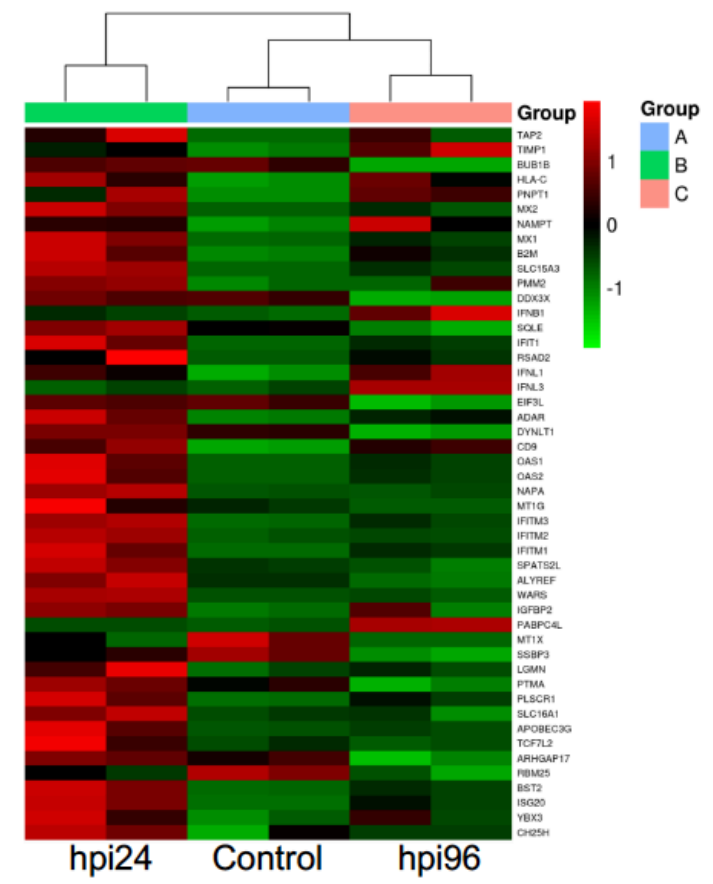

i47 Figure 4. RNA transcriptomic analysis of human brain organoids after WSN infection. (A) Hierarchical

;48 clustering heatmap of differentially expressed genes derived from a comparison of a group of control, hpi 96, 
;49 and hpi 24. (B) Venn diagram of upregulated and downregulated genes of brain organoids infected with WSN ;50 at 24 hpi and 96 hpi. (C) Top 10 enriched GO terms in brain organoids infected with WSN at 24 hpi and 96 ;51 hpi. (D) Top 10 upregulated and downregulated genes at 24 hpi and 96 hpi after WSN infection. (E) The ;52 upregulated and downregulated genes at 24 hpi and 96 hpi after WSN infection. (F) Heatmap of interferon ¡53 stimulating genes (ISGs). 
A

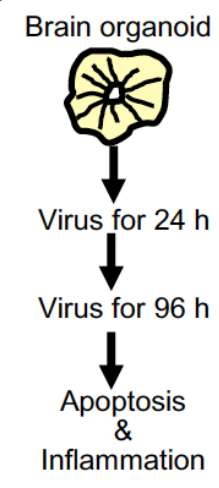

D

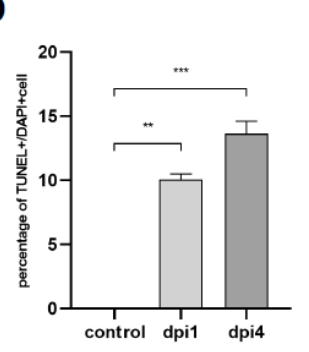

B

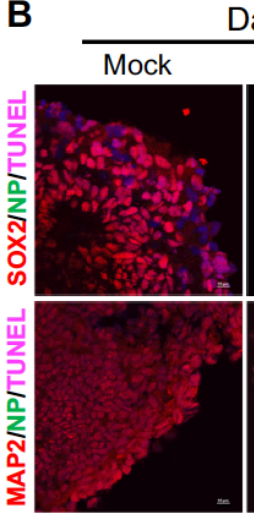

Day 30-organoid
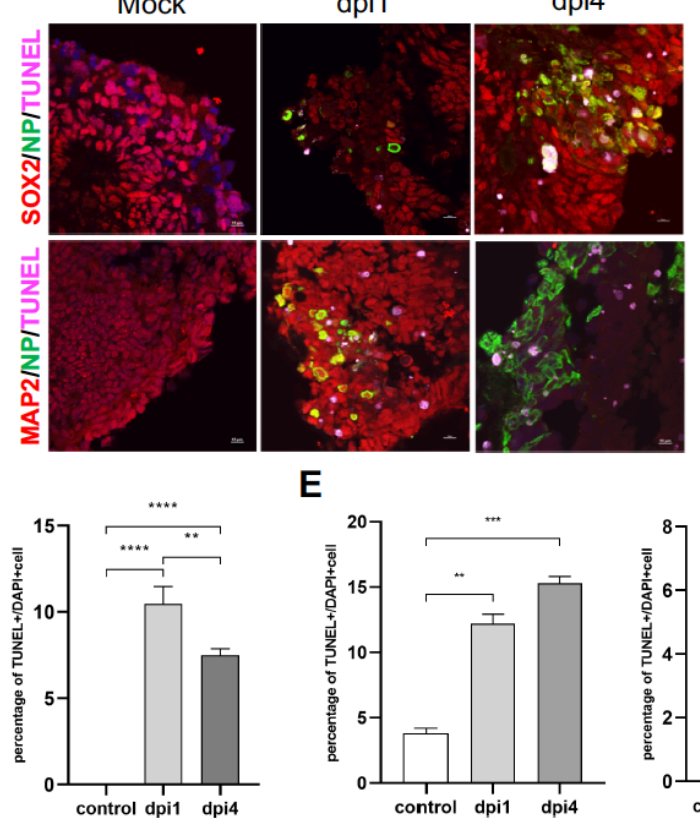

E

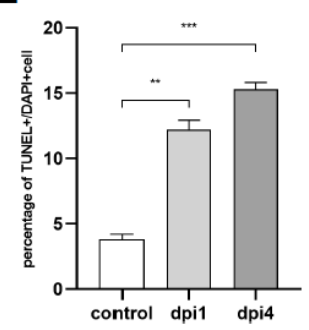

C

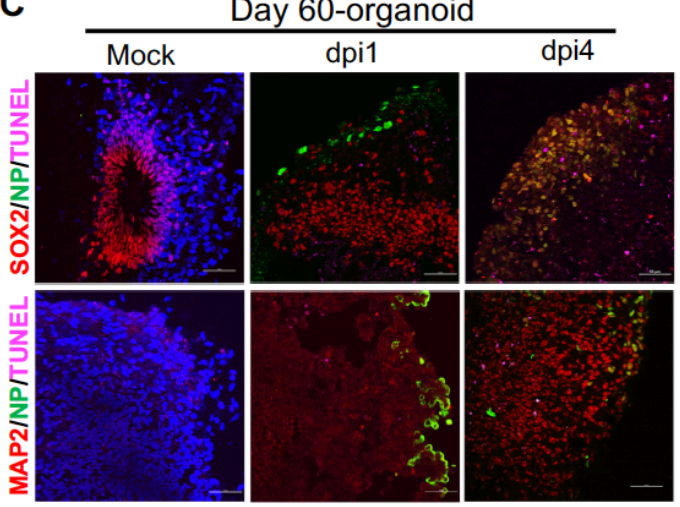

$\mathbf{F}$

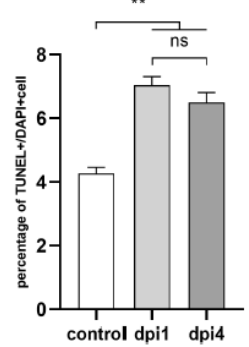

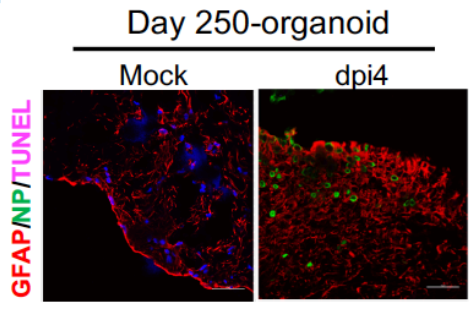

G
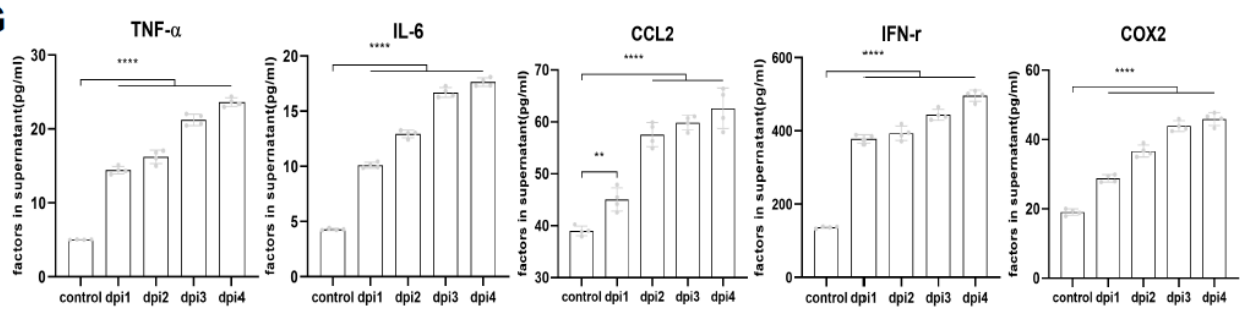

H
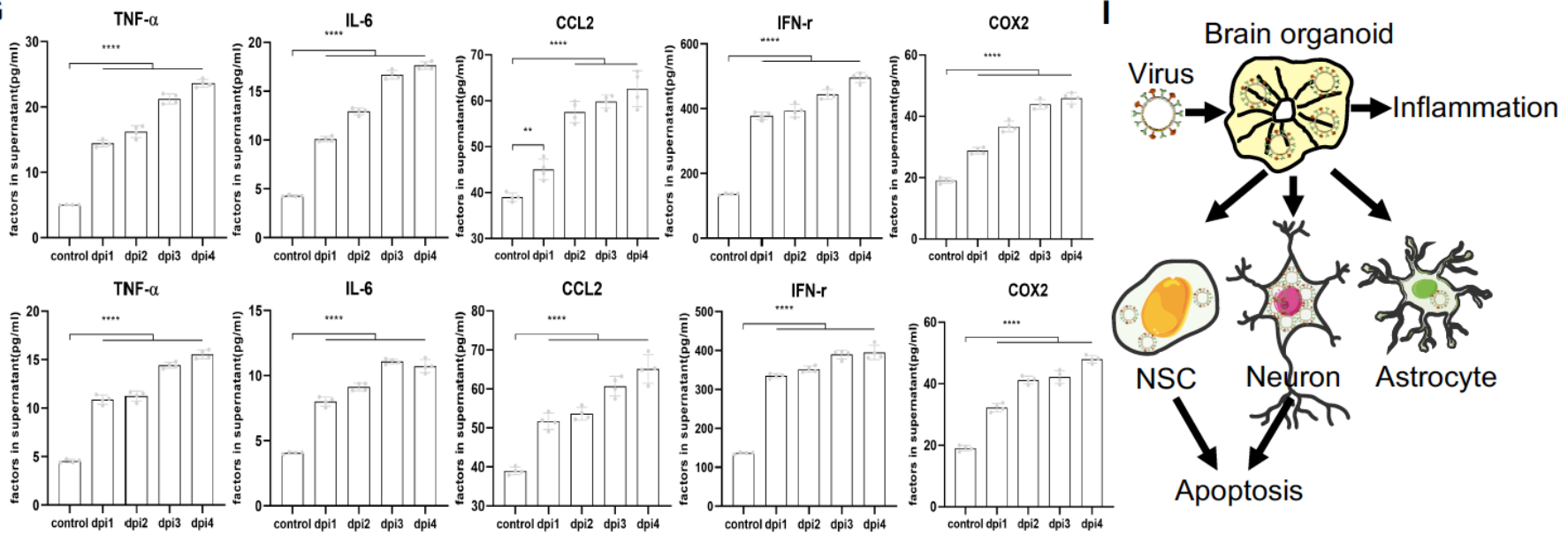

Figure 5. WSN induced cell apoptosis and inflammation in human brain organoids. (A-D) TUNEL

staining and quantification of positive cells in day-30 and day-60 brain organoids infected with WSN at 1 dpi and 4 dpi show a time-dependent increase, respectively. Scale bars, $10 \mu \mathrm{m}$. (E) TUNEL staining of GFAP+ astrocytes in day 250 brain organoids at 4 dpi. (F, G) Secreted inflammatory factors (e.g., TNF- $\alpha$, IL-6, CCL2, IFN- $\gamma$, and COX2) in day-30 (F) and day-60 (G) brain organoids at indicated infection timepoints. Scale bars, ;61 $100 \mu \mathrm{m}$. 
A

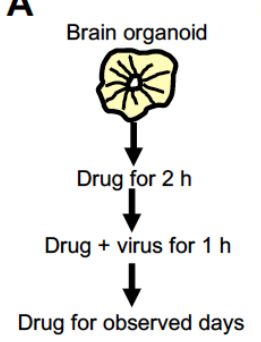

B

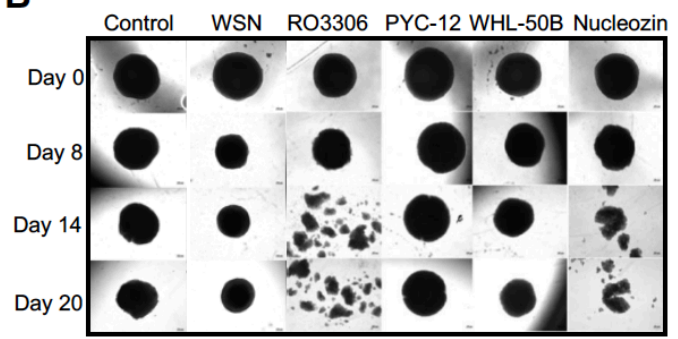

C

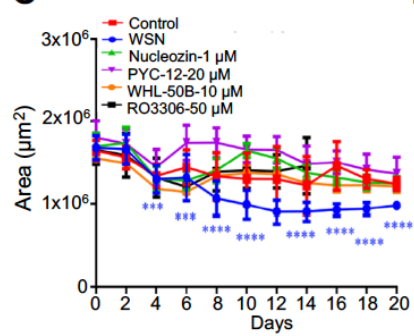

D

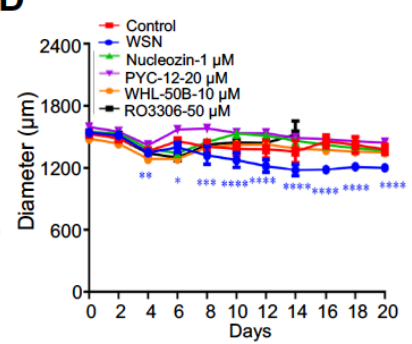

E

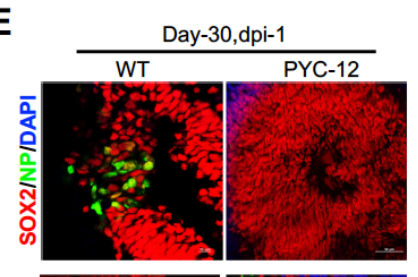

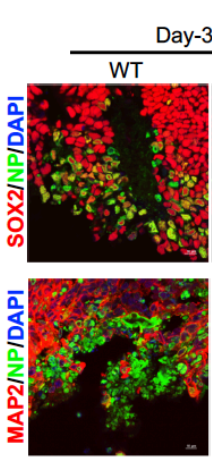

H
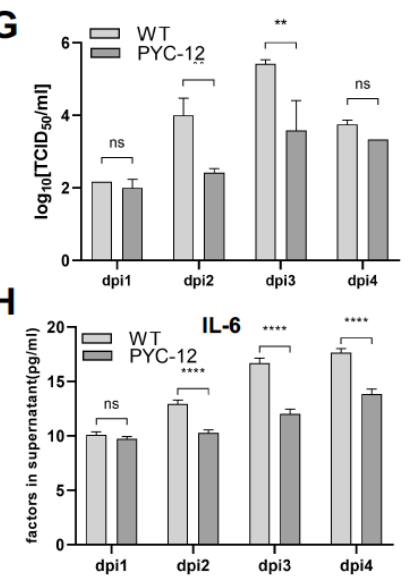
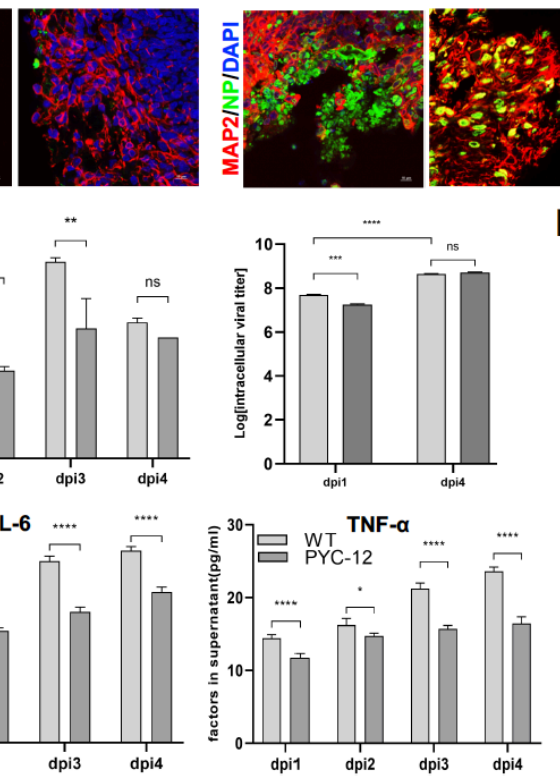

F
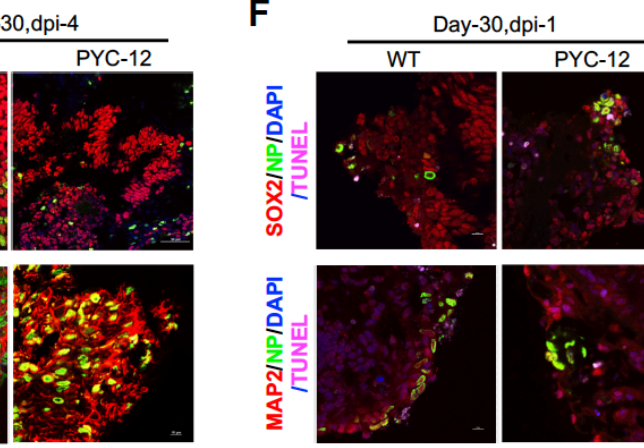

I
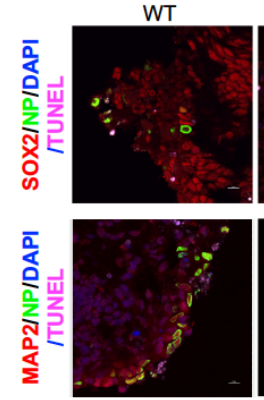
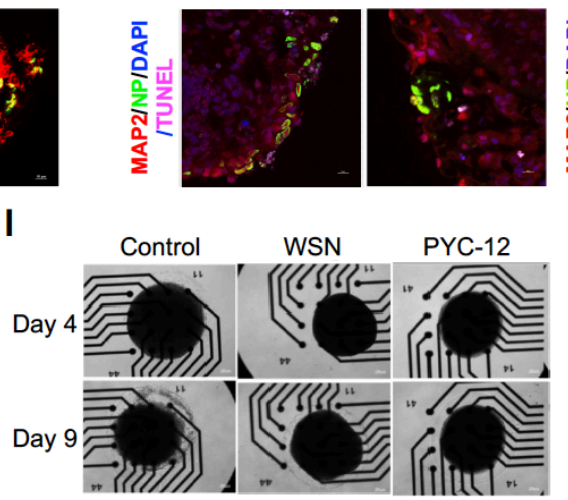

J 퐁

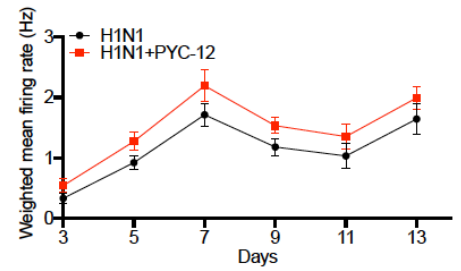

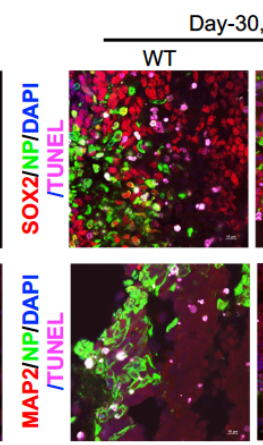

Day-30,dpi-4
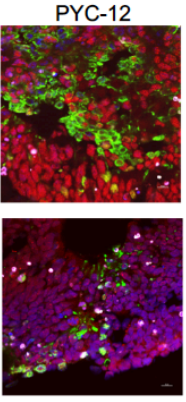

K

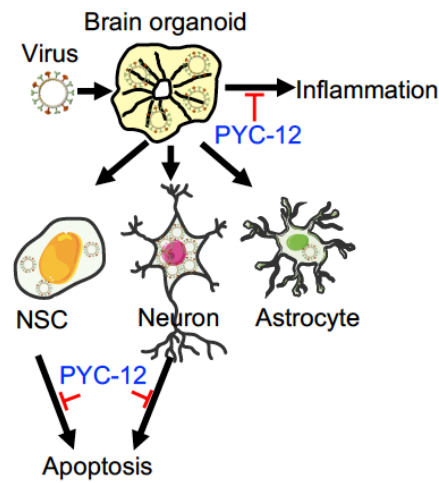

;64 the workflow for drug screening. (B) Representative bright field images of organoids cotreated with

;65 H1N1-WSN and several drugs at indicated time points. Nucleozin was used as a positive control. Scale bars,

;66 $50 \mu \mathrm{m}$. (C, D) Statistical analysis of area $\left(\mu \mathrm{m}^{2}\right)$ and diameter $(\mu \mathrm{m})$ of brain organoids infected with

;67 H1N1-WSN at indicated time points. (E) Immunostaining of neural stem cells and neurons in day 30 brain 
;69 staining of neural stem cells and neurons in day 30 brain organoids co-treated with WSN and PYC-12 for 1

;70 day and 4 days. Scale bars, $10 \mu \mathrm{m}$. (G) The viral titers of intracellular and supernatants from day 30 brain

;71 organoids co-treated with WSN and PYC-12. (H) The inflammatory factors release (IL-6 and TNF- $\alpha$ ) of

;72 day-30 brain organoids co-treated with WSN and PYC-12 at indicated infection timepoints. (I) The

;73 representative bright field images of microelectrode array (MEA) analysis of PYC-12 treated brain organoids.

;74 Scale bars, $50 \mu \mathrm{m}$. (J) Weighted mean firing rates (Hz) of brain organoids treated with H1N1 or cotreated with

;75 H1N1 and PYC-12 at indicated time points. (K) Schematic illustration of the antiviral strategy of PYC-12

¡76 through anti-apoptosis and anti-inflammation.

$; 77$ 


\section{Supporting information}

A

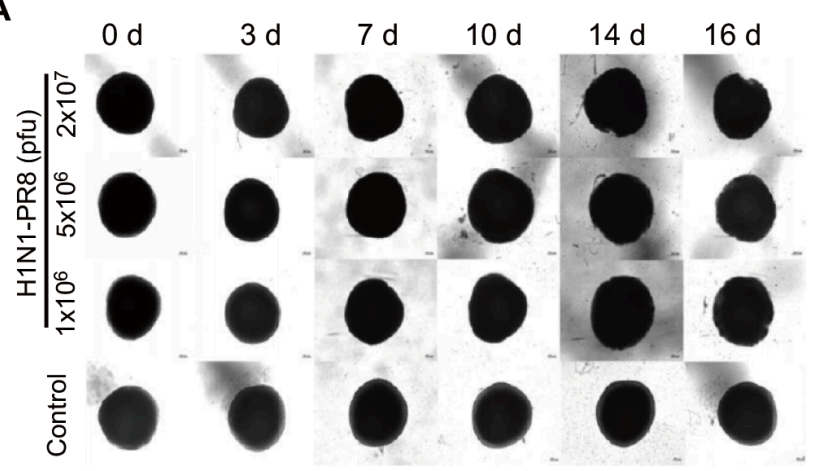

B

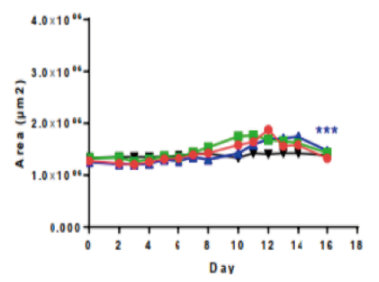

$; 79$

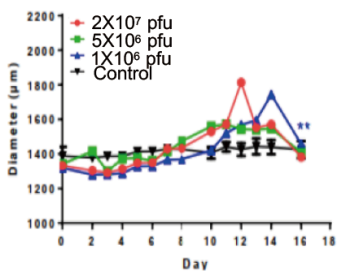

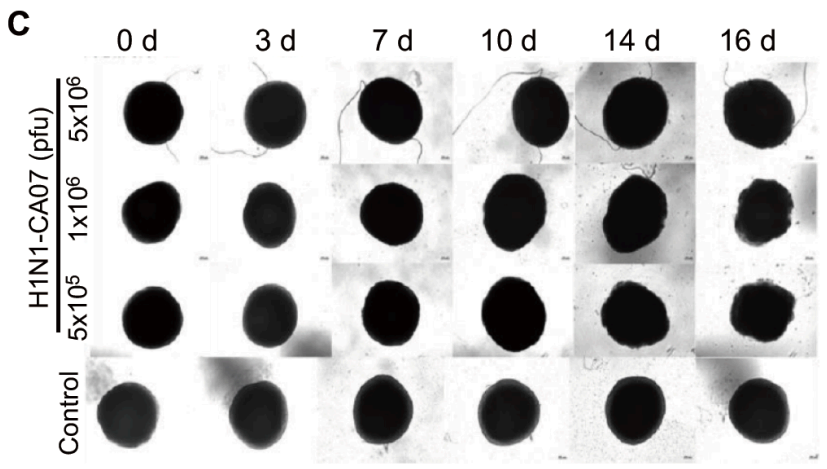

D

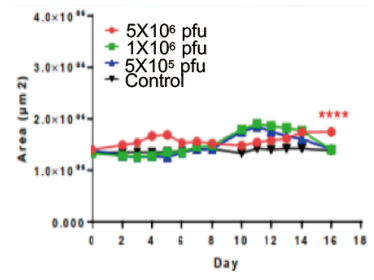

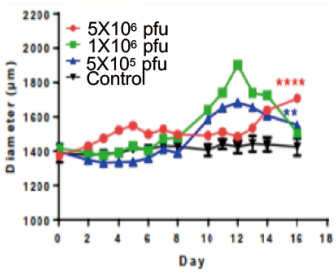

Figure S1. (A, C) Representative bright-field images of day 40-brain organoids infected with H1N1-PR8 or H1N1-CA07 at indicated time points. The infected concentrations for H1N1-PR8 were $1 \times 10^{6} \mathrm{pfu}, 5 \times 10^{6} \mathrm{pfu}$, and $2 \times 10^{7} \mathrm{pfu}$; for H1N1-CA07, they were $5 \times 10^{5} \mathrm{pfu}, 1 \times 10^{6} \mathrm{pfu}$, and $5 \times 10^{6} \mathrm{pfu}$. Scale bars, $50 \mu \mathrm{m}$. (B, D) Statistical analysis of area $\left(\mu \mathrm{m}^{2}\right)$ and diameter $(\mu \mathrm{m})$ of brain organoids infected with viruses at indicated time points. 
A

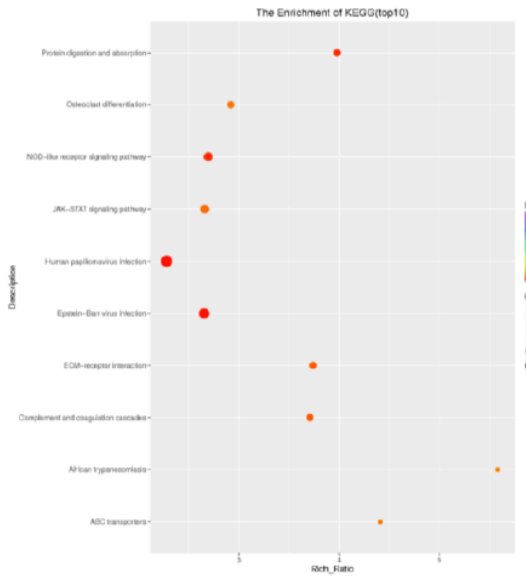

C

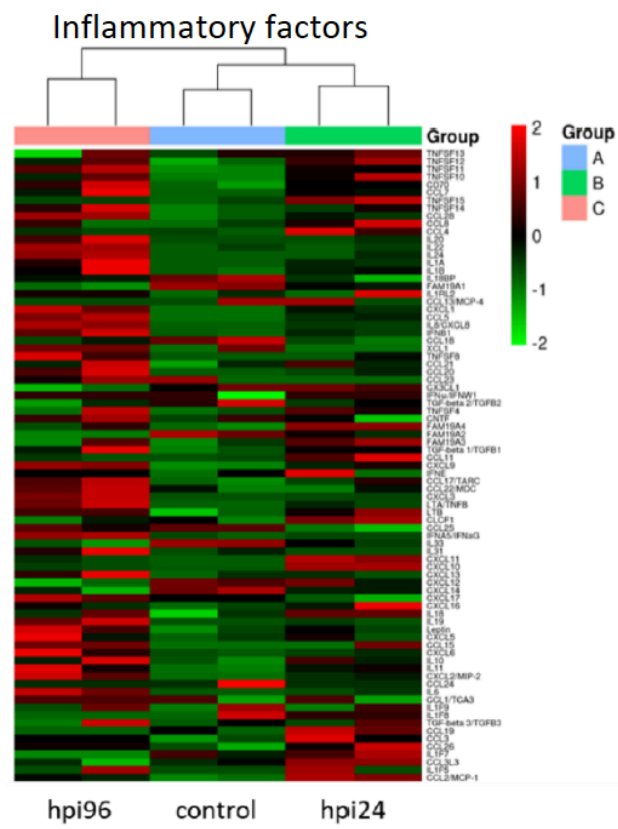

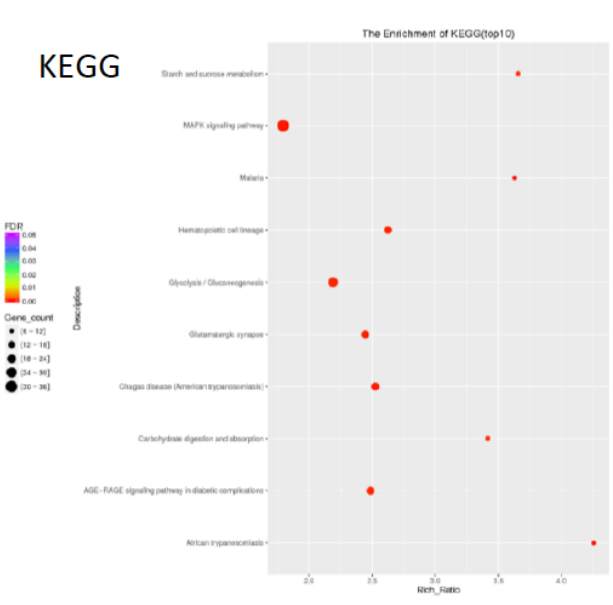

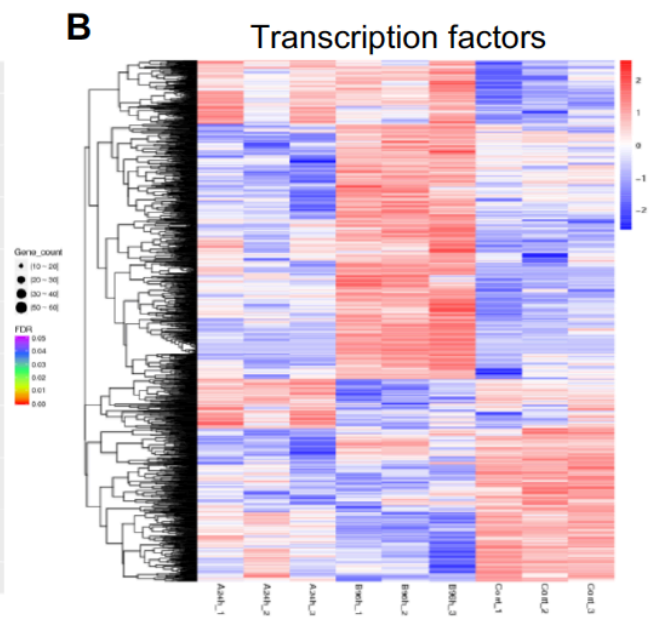

D

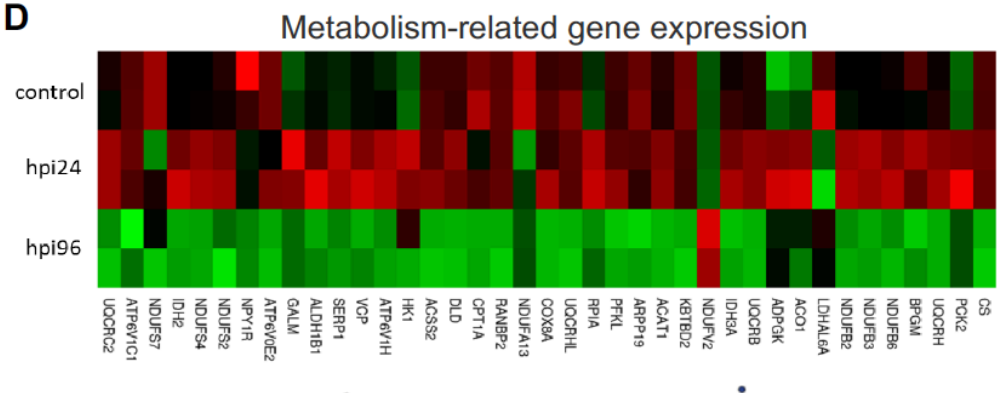

E

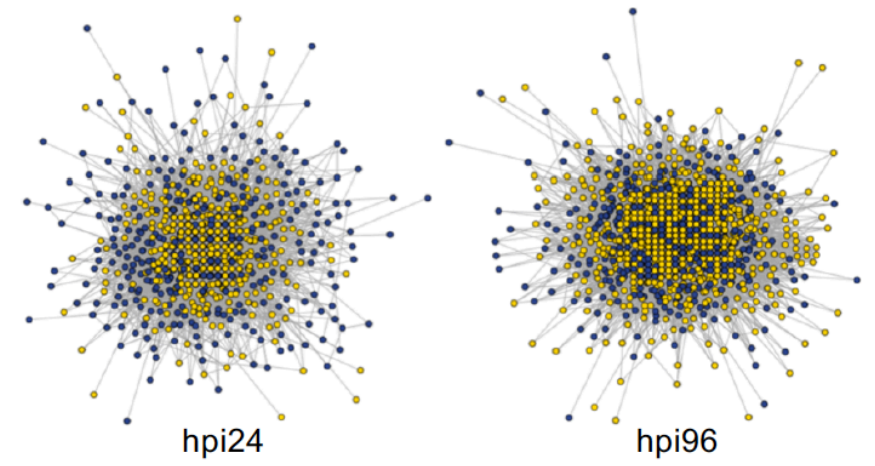

Figure S2. (A) The top 10 KEGG pathway enrichments of of brain organoids infected with WSN at 1 dpi and

4 dpi. (B) Heatmap of transcriptional factor expression in control, hpi24, and hpi96 groups. (C) Heatmap of

;89 inflammatory factor expression in control, hpi24, and hpi96 groups. (D) Heatmap of metabolic genes. (E)

;90 Protein-protein interaction network of WSN infected brain organoids at 24 hpi and 94 hpi. 


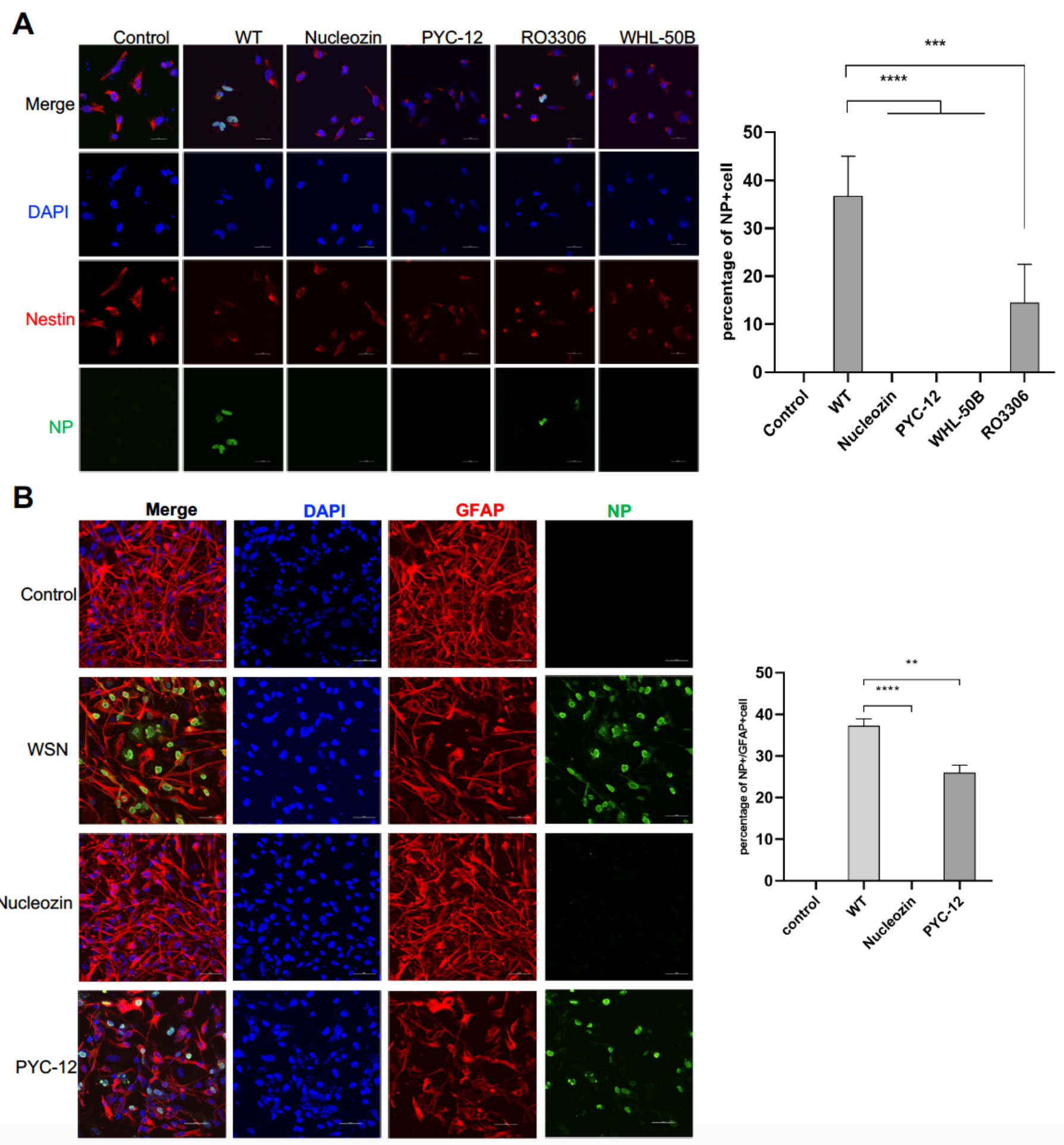

;93 Figure S3. Antiviral drug screening using NSCs and astrocytes. (A) Immunostaining and statistical ;94 analysis of neural stem cells treated with WSN, Nucleozin, PYC-12, RO3306, and WHL-50B. Non-treated 
;95 NSCs were used as a negative control. Scale bars, $20 \mu \mathrm{m}$. (B) Immunostaining and statistical analysis of

;96 astrocytes treated with WSN, nucleozin, and PYC-12. Non-treated astrocytes were used as a negative control.

¡97 Scale bars, $50 \mu \mathrm{m}$.

;98 
A

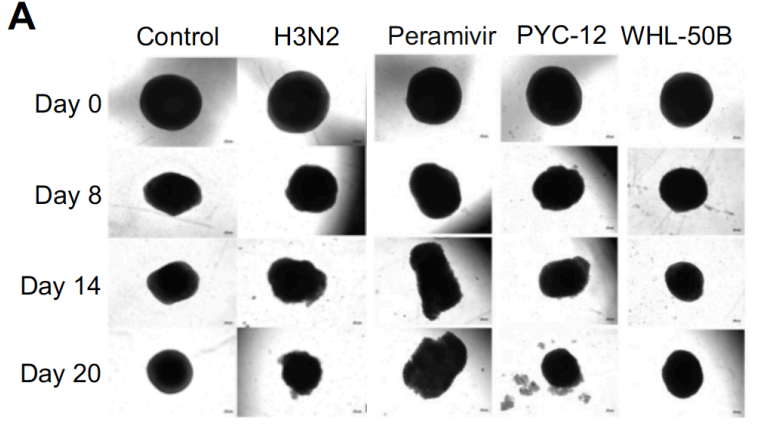

B

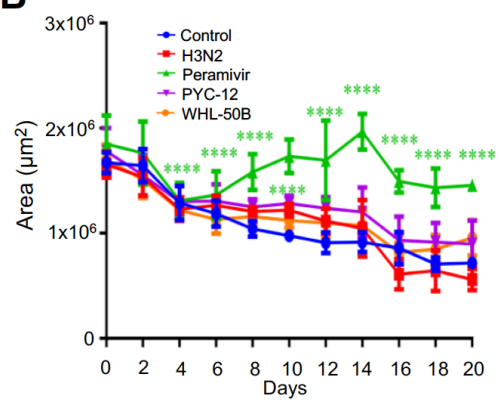

C

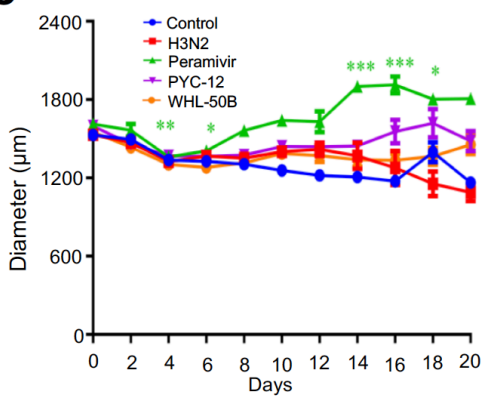

j00 Figure S4. Antiviral drug study of human brain organoids infected with H3N2-HKT68. (A)

j01 Phase-contrast images of brain organoids cotreated with H3N2-HKT68, peramivir, PYC-12, and WHL-50B at

$j 02$ indicated time points. Scale bars, $50 \mu \mathrm{m}$. $(\mathbf{B}, \mathbf{C})$ Statistical analysis of the area $\left(\mu \mathrm{m}^{2}\right)$ and diameter $(\mu \mathrm{m})$ of

j03 organoids cotreated with H3N2-HKT68 and different drugs at indicated time points. 

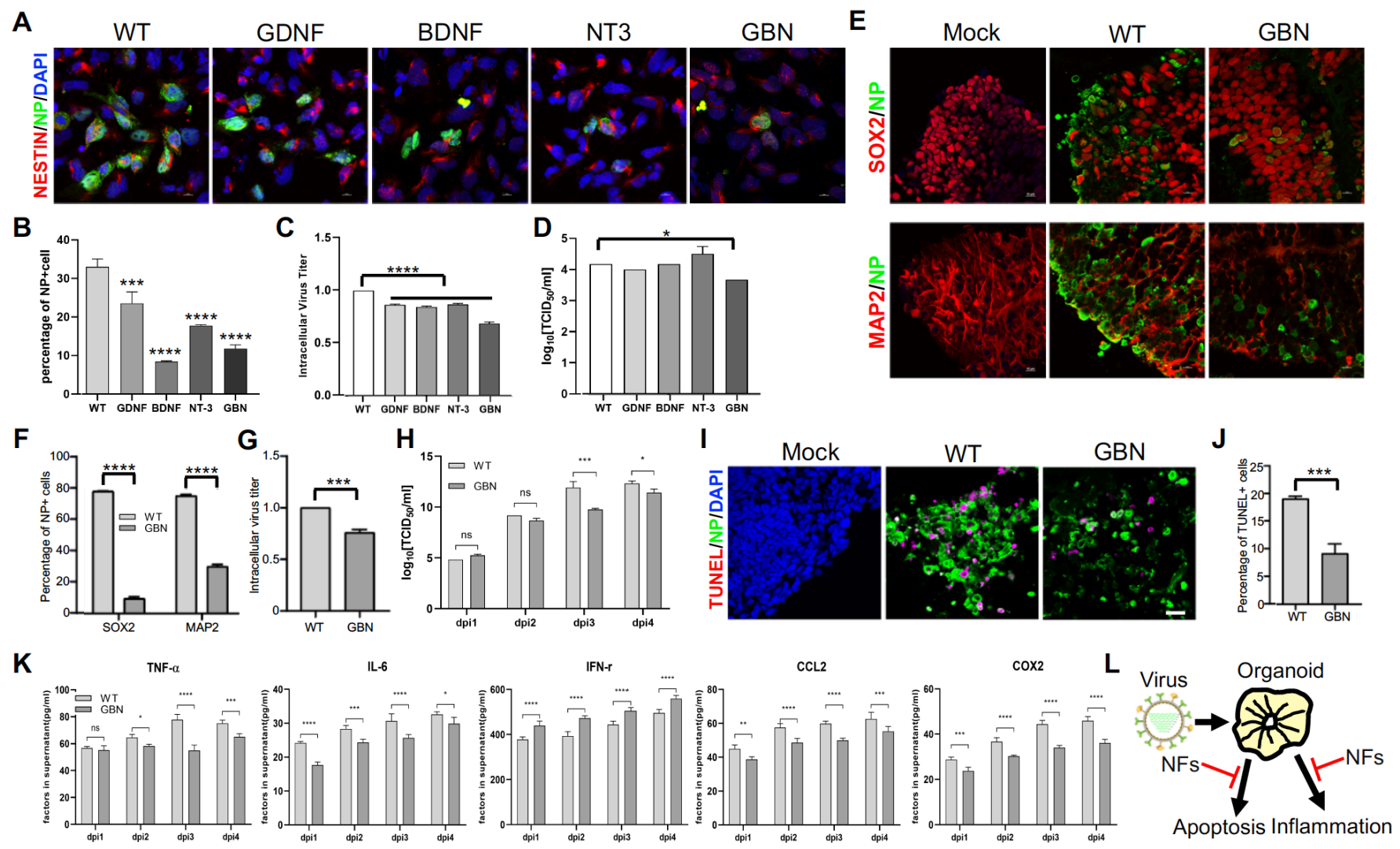

Figure S5. Neurotrophic factors inhibits WSN infection. (A) Immunostaining of NSCs treated with WSN

or different neurotrophic factors, including BDNF, GDNF and NT3. GBN denotes the combination of these organoids treated with WSN and GBN. Scale bar, $10 \mu \mathrm{m}$. (F) The percentage of NP+ cells after GBN

treatment compared to WSN infection. $(\mathbf{G}, \mathbf{H})$ The intracellular and extracellular virus titers after treatment. (I,

J) TUNEL staining and quantification of positive cells in day 30 brain organoids at 4 dpi. Scale bar, $50 \mu \mathrm{m}$.

$i 12$ (K) The secreted inflammatory factors (e.g., TNF- $\alpha$, IL-6, CCL2, IFN- $\gamma$, and COX2) from day 30 brain organoids at indicated infection timepoints. (L) Schematic illustration of antiviral methods produced through neurotrophic factors treatment. 

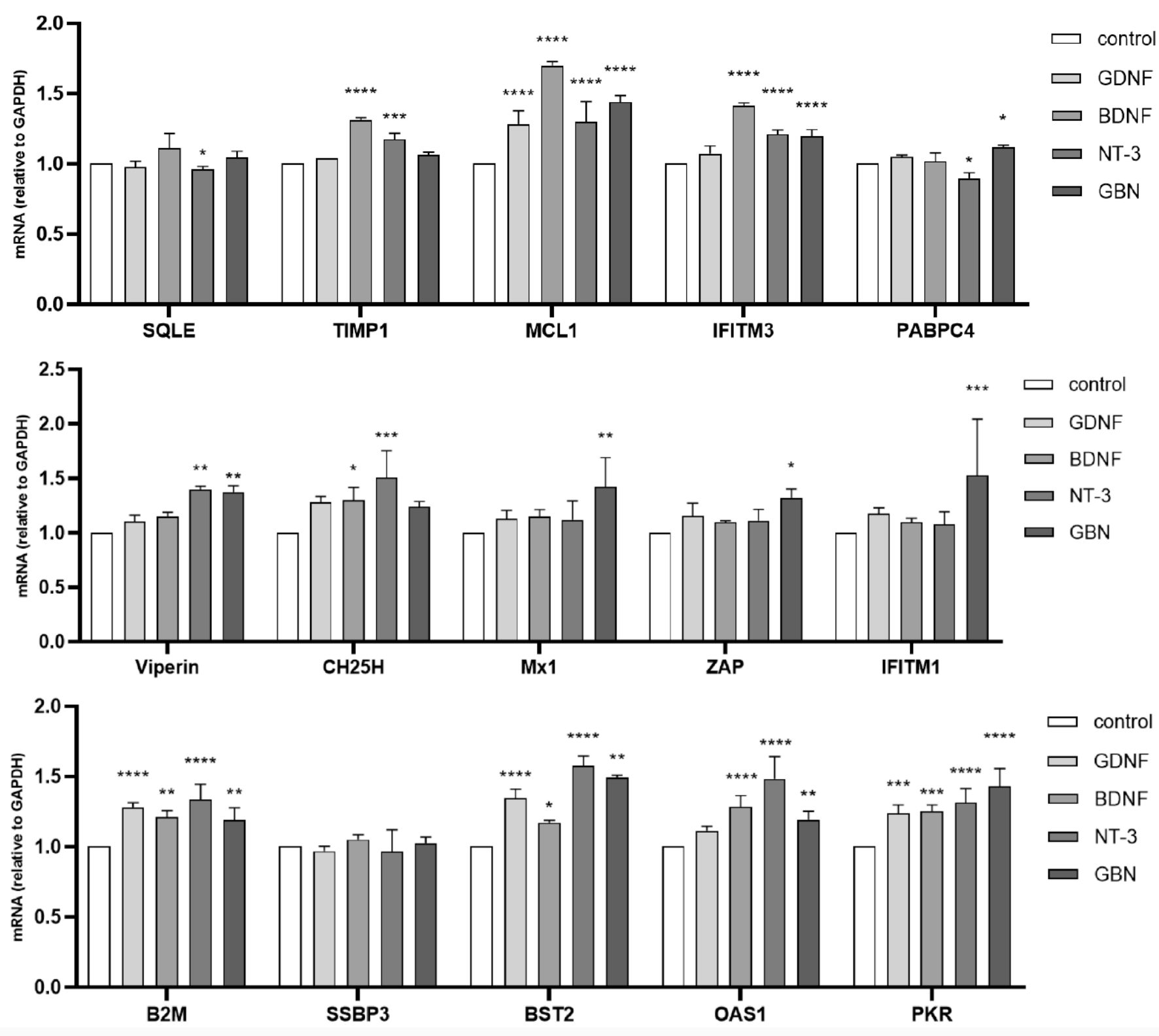

¡16 Figure S6. Insulin stimulating gene expression from organoids cotreated with WSN and neurotrophic factors

i17 by quantitative RT-PCR. 